\title{
ARTICLE Nod2 influences microbial resilience and susceptibility to colitis following antibiotic exposure
}

\author{
A. Goethel ${ }^{1,2}$, W. Turpin ${ }^{2,3}$, S. Rouquier ${ }^{3}$, G. Zanello ${ }^{3}$, S. J. Robertson ${ }^{1}$, C. J. Streutker ${ }^{4}$, D. J. Philpott ${ }^{1}$ and K. Croitoru $^{1,2,3,5}$
}

Inflammatory bowel disease (IBD) etiology involves genetic susceptibility, environmental triggers, and the gut microbiome. Antibiotic exposure is associated with IBD, both in early life and adulthood. Here, we investigated whether Nod2-deficiency influenced response of the gut microbiota to antibiotics and subsequent colitis susceptibility. Wild-type and Nod2 ${ }^{-/-}$littermate mice were treated with amoxicillin as adults or neonates, and fecal samples were collected for 16S rRNA sequencing. Five weeks after antibiotic exposure, dextran sulfate sodium (DSS) colitis was induced. Antibiotic treatment altered the microbiota of adult WT and $\mathrm{Nod}_{2}{ }^{-1-}$ mice, but recovery was delayed in Nod2 $2^{-1-}$ mice. Neonatal antibiotic treatment significantly changed the microbiota at weaning in WT and Nod2 $2^{-1-}$ littermates; however, Nod2 $2^{-1-}$ mice maintained reduced microbial diversity 14 days after cessation of antibiotics. Although treatment of adult mice did not influence susceptibility to colitis, neonatally treated Nod $2^{-1-}$ mice developed a more severe colitis. Moreover, the colitis phenotype was transferable through fecal transplantation into germ-free Nod $2^{-1-}$ recipients, and was associated with changes in intestinal T cells and the cytokine milieu following inflammation. These data demonstrate that neonatal antibiotic exposure has long-lasting influence on the microbiota and mucosal immunity, and may explain how NOD2 contributes to the risk of intestinal inflammation.

Mucosal Immunology (2019) 12:720-732; https://doi.org/10.1038/s41385-018-0128-y

\section{INTRODUCTION}

The mammalian gut typically harbors multiple types of microbes, including $\sim 4 \times 10^{13}$ bacteria, ${ }^{1}$ in a dynamic community whose structure depends on environmental and host genetic influences. ${ }^{2-6}$ Maintaining this symbiotic relationship is key to the balance between health and disease. Colonization of the gut microbiota during neonatal life influences the development of the mucosal immune system. ${ }^{7}$ Environmental perturbations during this critical window can alter the 'normal' development of the gut microbiota and the mucosal immune system, possibly increasing susceptibility to disease. Indeed, mouse and human studies suggest that neonatal antibiotic exposure is associated with an increased risk of inflammatory diseases, including asthma, ${ }^{8}$ obesity $^{9}$, and inflammatory bowel disease (IBD). ${ }^{10-12}$ This is supported by observations that early life and repeated antibiotic exposure significantly increases the risk for developing IBD. ${ }^{11,13}$

Given that Nod2 mutations are one of the most important genetic risks for the development of Crohn's disease, we investigated whether Nod2-deficiency altered the impact of antibiotic exposure on microbiota composition and susceptibility to colitis. We found that Nod2 influenced microbial resilience following adult and neonatal antibiotic treatment. Although the adult treatment did not alter colitis susceptibility, neonatal antibiotic treatment resulted in increased colitis severity in Nod $2^{-1-}$ mice. Moreover, transfer of the neonatal antibioticmicrobiota increased colitis severity in germ-free recipients. This phenotype was associated with altered cytokines and increased colonic Gata3 ${ }^{+}$Foxp3 $^{+}$regulatory T cells. This suggests that the sustained alterations of the gut microbiota following neonatal antibiotic exposure combined with the absence of Nod2 signaling during mucosal barrier breach results in exacerbated mucosal damage.

\section{RESULTS}

Nod2 genotype influences microbiota resilience after antibiotic exposure in adult mice

We first investigated how a single course of the broad-spectrum antibiotic, amoxicillin, altered the adult murine microbiota, and whether Nod2 genotype influenced this perturbation. Adult WT and $\mathrm{Nod}^{-1-}$ littermate mice were given amoxicillinsupplemented water for 7 days and stool samples were collected at day 0 (pre-treatment) and on day 7 (Fig. 1a). In order to further assess whether Nod 2 influenced the resilience or recovery of the microbiota following antibiotic exposure, stool was collected on day 21 , i.e., 14 days after stopping antibiotic-supplemented water. Each mouse was compared to its pre-treatment composition (day 0 ) and water-treated littermate controls. Using 16S rDNA sequencing, microbial diversity was evaluated for species richness (as measured by the Chao1 rarefaction index) and beta diversity (as measured by Bray-Curtis dissimilarity, which factors in presence/absence and relative abundance of operational taxonomic units).

As previously described, ${ }^{14}$ Nod2 genotype was not associated with any difference in the microbiota in terms of species richness, beta diversity, or composition at day 0 (Fig. $1 \mathrm{~b}-\mathrm{d}$ ), indicating that

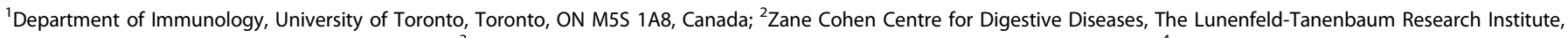

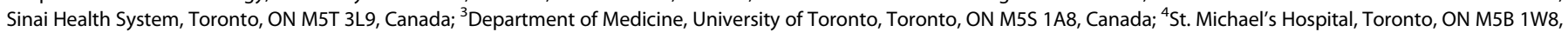
Canada and ${ }^{5}$ Division of Gastroenterology, Mount Sinai Hospital, Toronto, ON M5G 1X5, Canada Correspondence: K Croitoru (ken.croitoru@sinaihealthsystem.ca)
}

Received: 9 September 2018 Revised: 5 December 2018 Accepted: 16 December 2018 Published online: 16 January 2019 


\begin{tabular}{|c|c|c|}
\hline WT & Water & Water \\
\hline $\mathrm{Nod}_{2}{ }^{-I-}$ & Water & Water \\
\hline WT & Abx & Water \\
\hline $\mathrm{Nod}_{2}{ }^{-/-}$ & $A b x$ & Water \\
\hline & & \\
\hline Day & & \\
\hline Age (w) & & \\
\hline
\end{tabular}

b

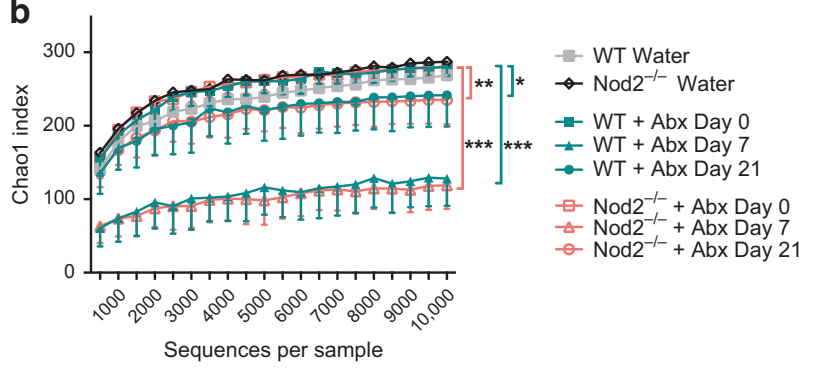

C
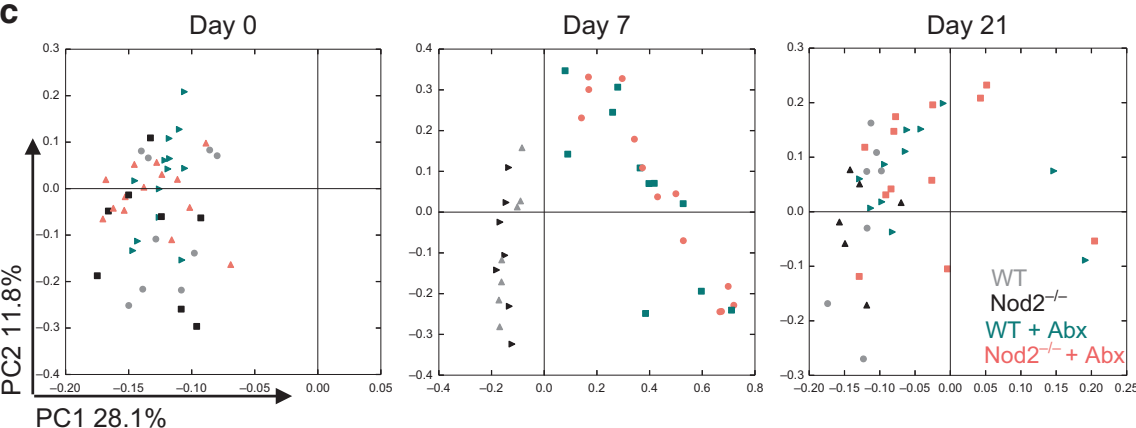

d
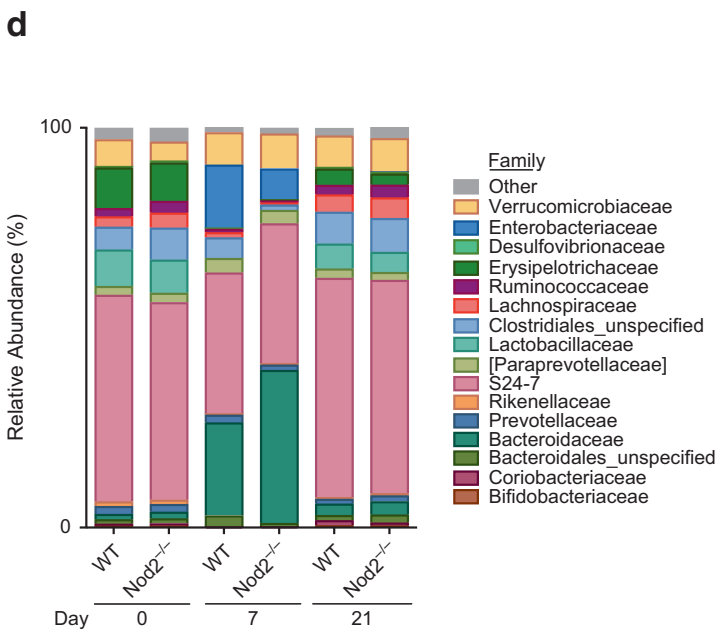

f

WT
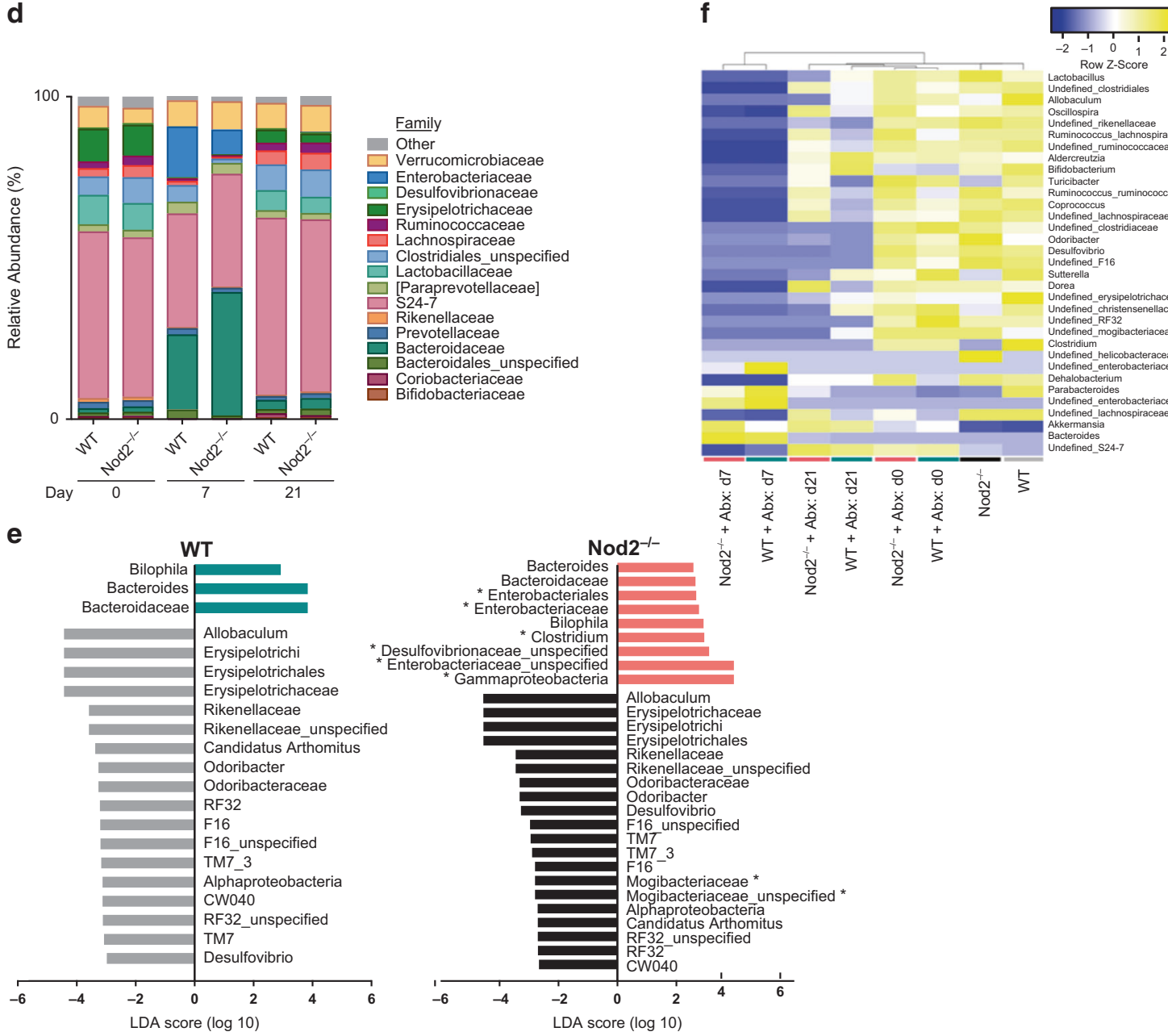

Lactobacillus Z-Score Undefined_lose
Allobaculum Oscillospira
Undefined_rikenellaceae Ruminocococus lactnospiracee
Undefined ruminococcaceae Indercreutziai Turicibacter Ruminococcus_ruminococcace Undefined_lachnospiracea Odoribacter
Desulfovibrio Undefined, Dorea Undefined_erysipeletrichaceae Undefined_CFristensenellacea Undefined_m Undefined_helicobacteraceae Underined_enterobacteriaceae
Dehalobacterium parabacteroides

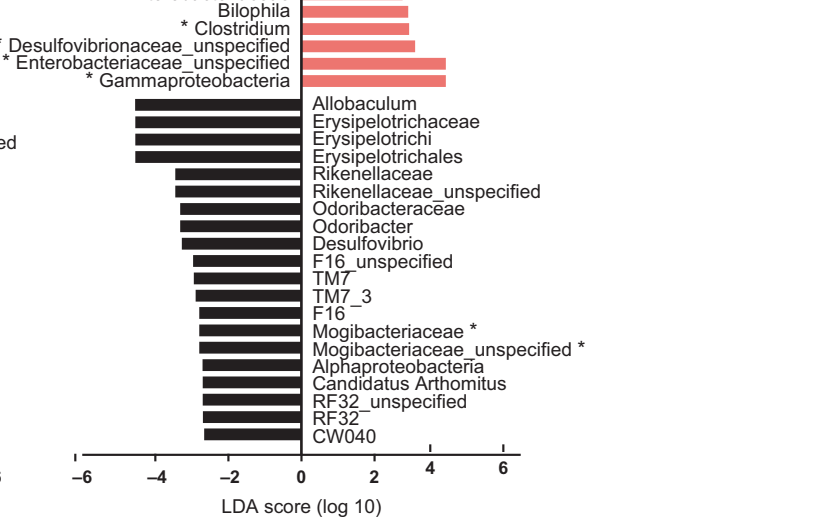


Fig. 1 Nod2 genotype influences microbiota resilience after antibiotic exposure in adult mice. a Experimental strategy. Starting at 8 weeks of age, littermate WT and Nod2 $2^{-1-}$ mice received either water or antibiotic (Abx) treatment for 7 days followed by 2 weeks of water to allow for microbial recovery. Arrows indicate time points of stool collection for sequencing. $\mathbf{b}$ Species richness at day 0,7 , and 21 as measured by the Chao1 rarefaction index. c Principal coordinates plot of Bray-Curtis dissimilarity for each time point. Each dot represents one mouse. (ANOSIM, $W T+A b x d 0$ vs. $7: R=0.6778, p=0.001 ; N o d 2^{-1-}+A b x d 0$ vs. $7: R=0.7922, p=0.001 ; W T+A b x d 0$ vs. $21: R=0.1494, p=0.013 ; N o d 2^{-1-}+A b x d 0$ vs. 21: $R=0.2144, p=0.001)$. d Changes in bacterial composition, at the Family level, over time in antibiotic-treated WT and Nod2 ${ }^{-1-}$ littermates. e Taxonomic differences identified by LEfSe before and after antibiotic treatment (day 0 vs. 21) in WT (left) and Nod2 ${ }^{-1-}$ (right) littermates. Taxa reduced on day 21 compared to day 0 have negative LDA scores; and taxa enriched on day 21 have positive LDA scores. Taxa that are different between antibiotic-treated WT and Nod2 $2^{-1-}$ mice are indicated (asterisk). f Heat map of significant bacterial taxa using Euclidean distance and centroid linkage clustering module (blue: low abundance; yellow: high abundance). Values were generated using the median of each group at each time point. Data are presented as mean \pm SD (b), or mean (d). ${ }^{*} p<0.05,{ }^{* *} p<0.01,{ }^{* * *} p<0.001 ;$ by paired Student's $t$-test (b), ANOSIM (c), or Kruskal-Wallis (d). (WT $n=7, \operatorname{Nod} 2^{-1-} n=7$, WT + Abx $n=11$, Nod2 ${ }^{-1-}+$ Abx $n=13$. Data are from three independent experiments.)

microbiota of both adult WT and Nod2-/- mice was significantly altered by a seven-day course of amoxicillin treatment.

Recovery of the microbiota following antibiotic treatment was assessed on day 21. Antibiotic-treated WT and Nod2 $2^{-1-}$ mice maintained a significantly different microbiota than pre-treatment composition (WT: $R=0.1494, p=0.013$, Nod2 ${ }^{-/-}: R=0.2144, p=$ 0.001 ; Fig. 1c). Moreover, species richness at day 21 was significantly lower than pre-treatment richness for both genotypes (Fig. 1b). In order to further determine which bacterial taxa contributed to the differences both statistically and biologically, we utilized linear discriminant analysis (LDA) effect size (LEfSe) analysis. ${ }^{15}$ LEfSe analysis comparing the microbiota at day 0 vs. 21 of antibiotic-treated WT mice identified taxa that differed in abundance following antibiotic treatment (Fig. 1e, left panel). Taxa that were reduced or absent at day 21 compared to day 0 included Erysipelotrichaceae, Rikenellaceae, and Odoribacteraceae. Taxa enriched at day 21 compared to day 0 included Bacteroides and Bilophila. These compositional changes were induced by the antibiotic treatment and failed to resolve by day 21. Many of these taxa changes were shared in both antibiotictreated WT and Nod2 $2^{-1-}$ mice, however, there were differences between the genotypes. LEfSe analysis of antibiotic-treated Nod $2^{-1-}$ mice further identified several taxa that differed between day 0 and 21 only in Nod2-deficient mice (Fig. 1e, right panel), including expansion of Gammaproteobacteria and Desulfovibrionaceae on day 21. Moreover, at day 21, antibiotic-treated Nod2-1mice were enriched for the Gammaproteobacteria Enterobacteriaceae and had lost the family of Mogibacteriaceae compared to WT mice. These taxonomic differences due to antibiotic treatment were further highlighted using Euclidean distance and centroid linkage clustering analysis (Fig. 1f). These data suggest that Nod2 influenced both the changes in microbes in response to antibiotic treatment, and the resilience or recovery of the microbiota in adult mice.

Nod2 influences microbial response to antibiotic treatment during the neonatal period

We next investigated the impact of Nod2 genotype on the microbiota response to antibiotic treatment during the neonatal period (from birth until weaning-day 21 after birth), since environmental exposures significantly influence development of the intestinal microbiota in early life. ${ }^{16}$ Following birth, dams and newborn pups received water supplemented with amoxicillin, provided ad libitum until the age of weaning (Fig. 2a). Microbiota composition was assessed in stool samples collected at weaning (day 21). In order to monitor the maturation and recovery of the microbiota, stool samples were also collected on day 35 (14 days after weaning and cessation of antibiotic treatment). As observed in the adult treatment, neonatal antibiotic exposure resulted in a significant shift of the microbial community composition away from that in water-treated litters at day 21 (Supplementary Figure $1 \mathrm{~A}$ ). Although there was minimal variability within watertreated litters, there was significantly greater variability within antibiotic-treated litters, as measured by Bray-Curtis dissimilarity distance (Supplementary Figure 1B). The distance between water and antibiotic-treated litters was the greatest, emphasizing the community perturbation induced by antibiotic treatment. Thus, early life antibiotic exposure significantly altered the gut microbiota at weaning.

We then examined the influence of Nod2 genotype on the response of gut microbiota to early life antibiotic exposure. Diversity, as assessed using the Chao1 index of OTU richness, at weaning was not different between water-treated WT and Nod $2^{-1-}$ littermates; however, community richness was significantly diminished in antibiotic-treated WT and $\mathrm{Nod}^{-1-}$ mice at weaning (Fig. 2b). Moreover, the microbial communities of antibiotic-treated WT and Nod2 $2^{-1-}$ littermates were significantly different from that of their water-treated genotype-matched controls on day 21 (WT vs. WT+Abx: $R=0.5229, p=0.001$; Nod2 $2^{-1-}$ vs. Nod2 $2^{-1-}+$ Abx: $R=0.4929, \quad p=0.001$; Fig. 2c). Antibiotic treatment reduced the relative abundance of Bifidobacteriaceae, Lactobacillaceae, Lachnospiraceae, and Erysipelotrichaceae, and induced a bloom of Bacteroidaceae in both WT and Nod2 $2^{-1-}$ mice (Fig. 2d, Supplementary Table 1-3). Several important differences were observed in the response to antibiotic treatment at weaning between $\mathrm{Nod}^{-1-}$ and WT littermates. Specifically, antibiotic-treated $N o d 2^{-1-}$ mice experienced a decrease of Coriobacteriaceae, Rikenellaceae, S24-7, Paraprevotellaceae, Ruminococcaceae, and a bloom of Enterobacteriaceae compared to Nod2 $2^{-1-}$ water-treated controls (Fig. 2d, Supplementary Table 3); antibiotic-treated WT mice only had elevated relative abundance of Verrucomicrobiaceae compared to watertreated WT controls (a change not observed in the antibiotictreated $\mathrm{Nod2}^{-1-}$ mice) (Supplementary Table 2). These data suggest a role for Nod2 in shaping the response of certain bacterial taxa to antibiotic perturbation.

Absence of Nod2 signaling influences the resilience of gut microbiota two weeks after neonatal antibiotic exposure We sought to determine whether neonatal antibiotic-induced changes in the microbiota resolved 14 days after weaning. Antibiotic treatment was stopped at weaning and stool samples were collected on day 35 after birth (14 days post weaning) (Fig. 2a). No differences in microbiota diversity or composition were observed between water-treated WT and Nod2 ${ }^{-1-}$ littermates $(R=0.0199, p=0.250$, Supplementary Table 4$)$, or between water and antibiotic-treated WT mice on day $35(R=0.1322, p=$ 0.091; Fig. 2c, Supplementary Table 5), suggesting that the gut microbiota of WT mice had recovered 14 days after cessation of antibiotics.

In contrast, the microbiota in antibiotic-treated $\mathrm{Nod}^{-1-}$ mice failed to recover, in that there was a significantly different community compared to water-treated Nod $2^{-1-}$ controls $(R=$ $0.3726, p=0.001$; Fig. 2c). This was evident in the Chao1 index comparisons with antibiotic-treated $\mathrm{Nod}^{-1-}$ mice having lower richness (Fig. 2b) than their water-treated controls at day 35. Specifically, Nod2 $2^{-1-}$ mice that received antibiotic treatment had persistently increased relative abundance of 
a

\begin{tabular}{|c|c|c|}
\hline $\begin{array}{r}\text { Age }(w) \\
\text { Day }\end{array}$ & & \\
\hline Water-treated litter & Water & Water \\
\hline Antibiotic-treated litter & $A b x$ & Water \\
\hline
\end{tabular}

b

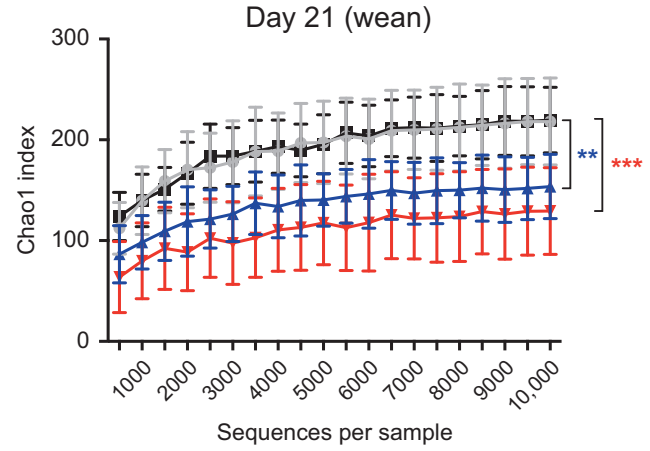

C

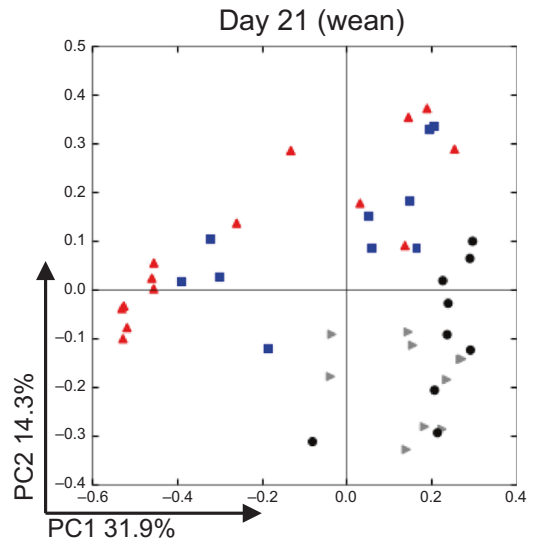

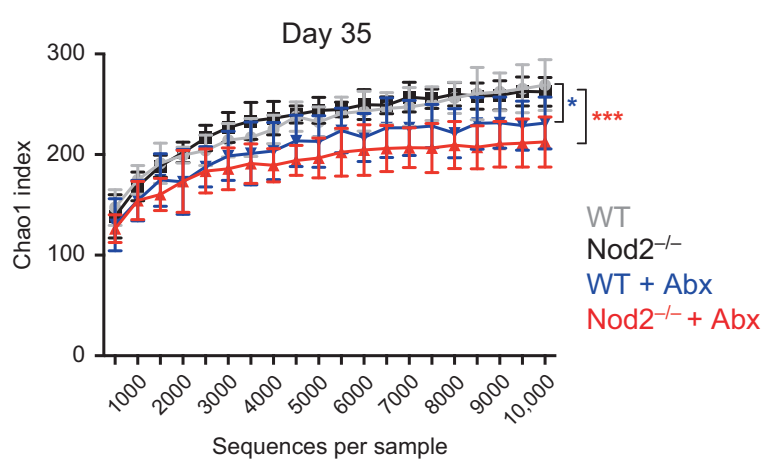

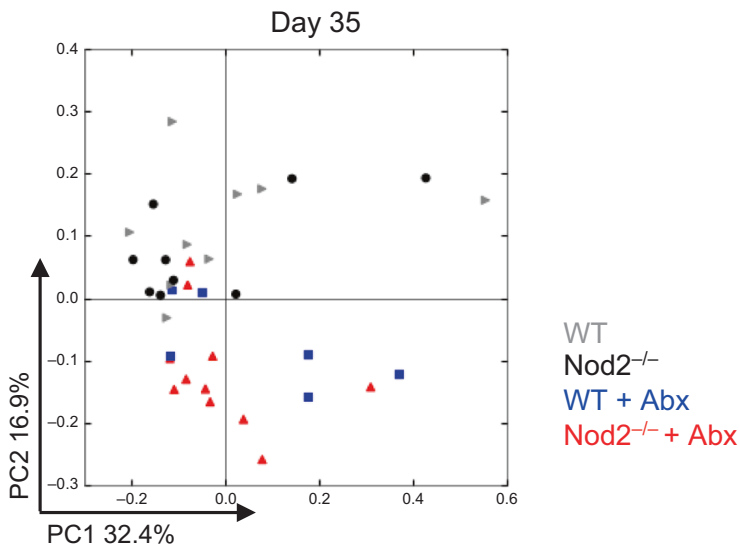

d

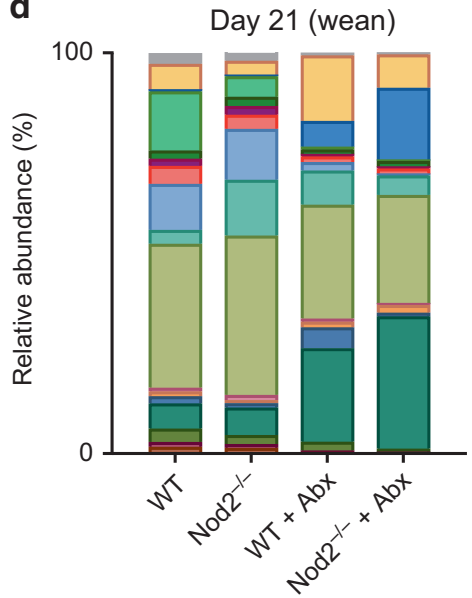

Family

Other

Verrucomicrobiaceae*

Enterobacteriaceae \#

Erysipelotrichaceae * \#

Ruminococcaceae \#

Lachnospiraceae* \#

Clostridiales unspecified

Lactobacillaceae* \#

[Paraprevotellaceae]\#

S24-7 \#

Rikenellaceae \#

Prevotellaceae

Porphyromonadaceae

Bacteroidaceae * \#

Bacteroidales unspecified \#

Coriobacteriaceae \#

Bifidobacteriaceae * \#

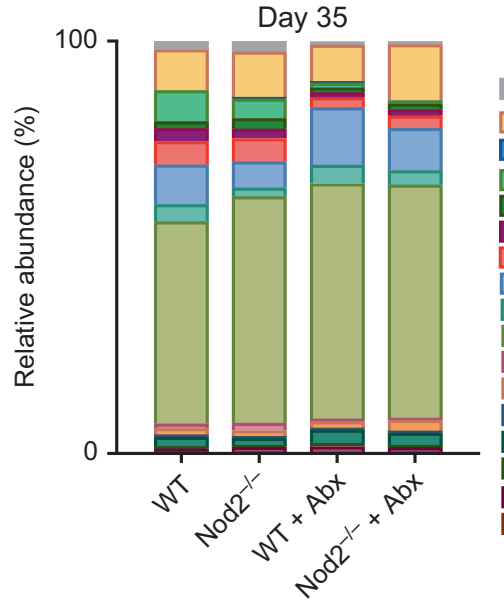

Family

Other

Verrucomicrobiaceae

Enterobacteriaceae

Erysipelotrichaceae\#

Ruminococcaceae

Lachnospiraceae

Clostridiales_unspecified

Lactobacillaceae

[Paraprevotellaceae]

S24-7

Rikenellaceae \#

Prevotellaceae

Porphyromonadaceae\#

Bacteroidaceae

Bacteroidales_unspecified

Coriobacteriaceae

Bifidobacteriaceae\#

Fig. 2 Nod2 influences responses to antibiotic treatment during the neonatal period. a Experimental outline. WT and Nod2 ${ }^{-/-}$littermates were divided into two treatment groups: water-treated (litters received only non-acidified control water from day 0 (following birth) throughout the study); or antibiotic-treated (litters received treatment with non-acidified water supplemented with amoxicillin (Abx) at $200 \mathrm{mg} / \mathrm{L}$ following birth until weaning (day 21). Weaned pups received control water until endpoint.). Arrows indicate stool collection time points. b Species richness as measured by the Chao1 index on day 21 and 35. c Principal coordinates plot of Bray-Curtis dissimilarity on day 21 and 35 . Each dot represents the stool microbiome of one mouse. (ANOSIM, Day 21: $W T$ vs. Nod $2^{-1-}: R=0.1796, p=0.019, W T$ vs. $W T+A b x: R=0.5229, p=0.001$; Nod2 $2^{-I-}$ vs. Nod2 ${ }^{-1-}+A b x: R=0.4929, p=0.001 ; W T+A b x$ vs. Nod2 ${ }^{-1-}+A b x: R=0.0906, p=0.084 ;$ Day $35: W T$ vs. Nod2 ${ }^{-1-}: R=0.0199, p=$

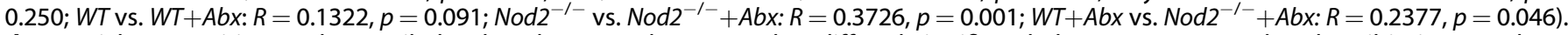
d Bacterial composition at the Family level at day 21 and 35. Taxa that differed significantly between untreated and antibiotic-treated WT (asterisk), or Nod2 $2^{-1-}$ (hash) mice are indicated (false-discovery rate corrected $p$-value $<0.05$ ). Data are presented as mean \pm SD (b) or mean (d). ${ }^{*} p<0.05,{ }^{* *} p<0.01,{ }^{* * *} p<0.001$; by paired Student's $t$-test (b) or Kruskal-Wallis (d). $(n=9-14$ mice per genotype per treatment. Data are from four untreated litters and five antibiotic-treated litters) 


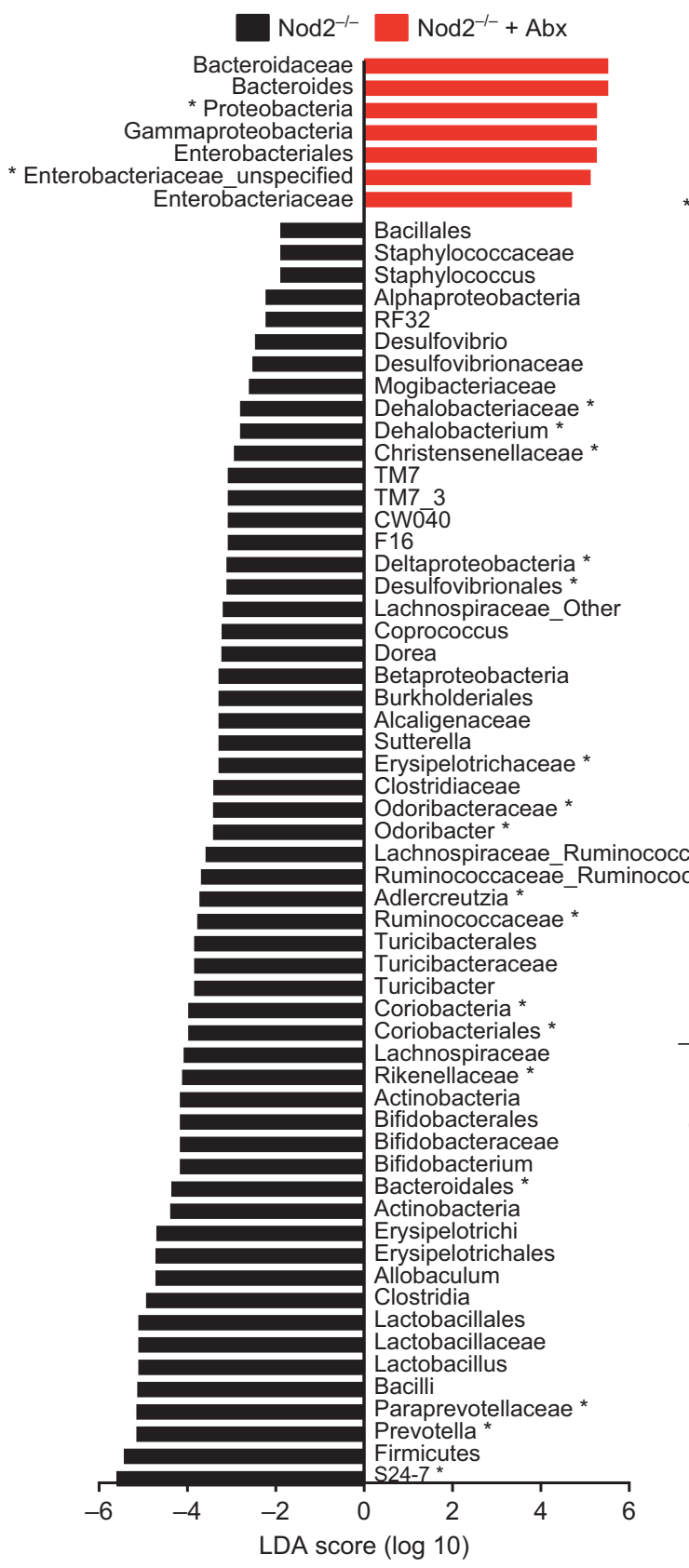

b

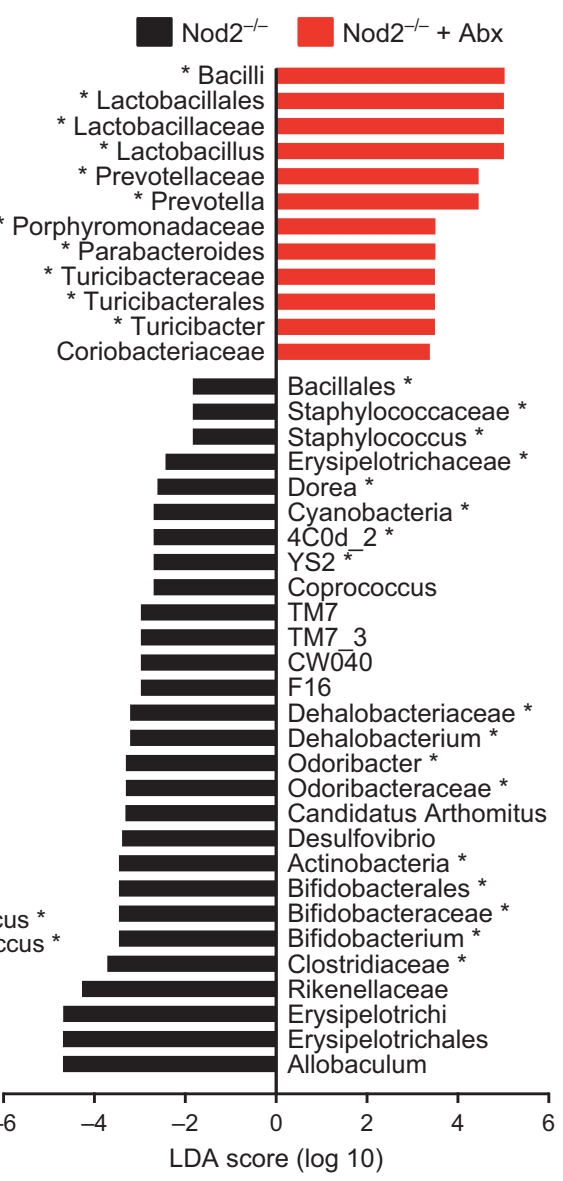

C

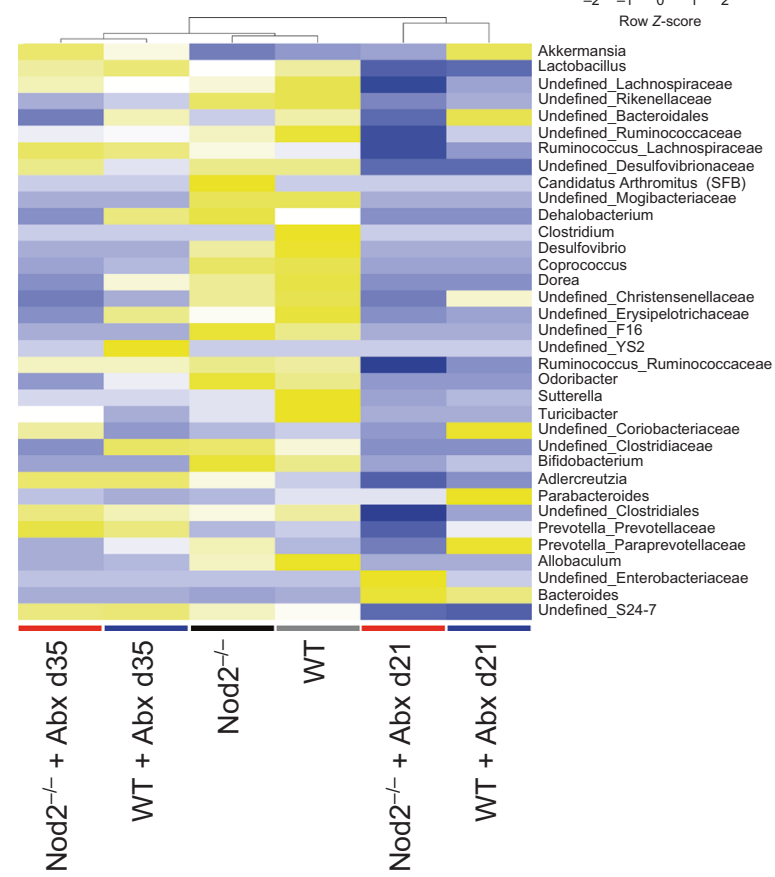

Fig. 3 Antibiotic-altered composition differs between $\mathrm{Nod}^{-1-}$ and WT littermates in neonatal period. Taxonomic differences were identified by linear discriminant analysis (LDA) coupled with effect size (LEfSe). a Taxonomic differences between water vs. antibiotic-treated Nod2 ${ }^{-\prime-}$ mice at day 21. Taxa enriched in water-treated are shown in black; taxa enriched in antibiotic-treated are red. Taxa that differed between WT and Nod2 $2^{-1-}$ mice are indicated (asterisk). b Taxonomic differences between water vs. antibiotic-treated Nod $2^{-1-}$ mice at day 35 . Taxa enriched in water-treated are shown in black; taxa enriched in antibiotic-treated are red. Taxa that differed between WT and Nod2 ${ }^{-1-}$ mice are indicated (asterisk). c Heat map of significant bacterial taxa using Euclidean distance and centroid linkage clustering module (blue: low abundance; yellow: high abundance). Values were generated using the median of each group at each time point 
Porphyromonadaceae, and decreased levels of Bifidobacteriaceae, Rikenellaceae, and Erysipelotrichaceae compared to their watertreated controls (Fig. 2d, Supplementary Table 6).

In order to better describe the strength and direction of the taxonomic changes induced by neonatal antibiotic treatment and the influence of Nod2 genotype, LEfSe analysis was performed. As described above, all of the antibiotic-induced differences between water and antibiotic-treated WT mice at weaning were also observed in $\mathrm{Nod}^{-/-}$mice, including a reduction in Lactobacillaceae and Bifidobacteraceae (Fig. 3a). On the other hand, Nod2 ${ }^{-1-}$ mice showed additional changes in several bacterial families, including decreased relative abundance of Rikenellaceae, S24-7 and Christensenellaceae (Fig. 3a, indicated with $a{ }^{*}$ ). Direct comparison of antibiotic-treated WT and $\mathrm{Nod}^{-/-}$mice further highlighted microbial differences related to host genetics, primarily expansion of Flexispira and an unspecified genus of
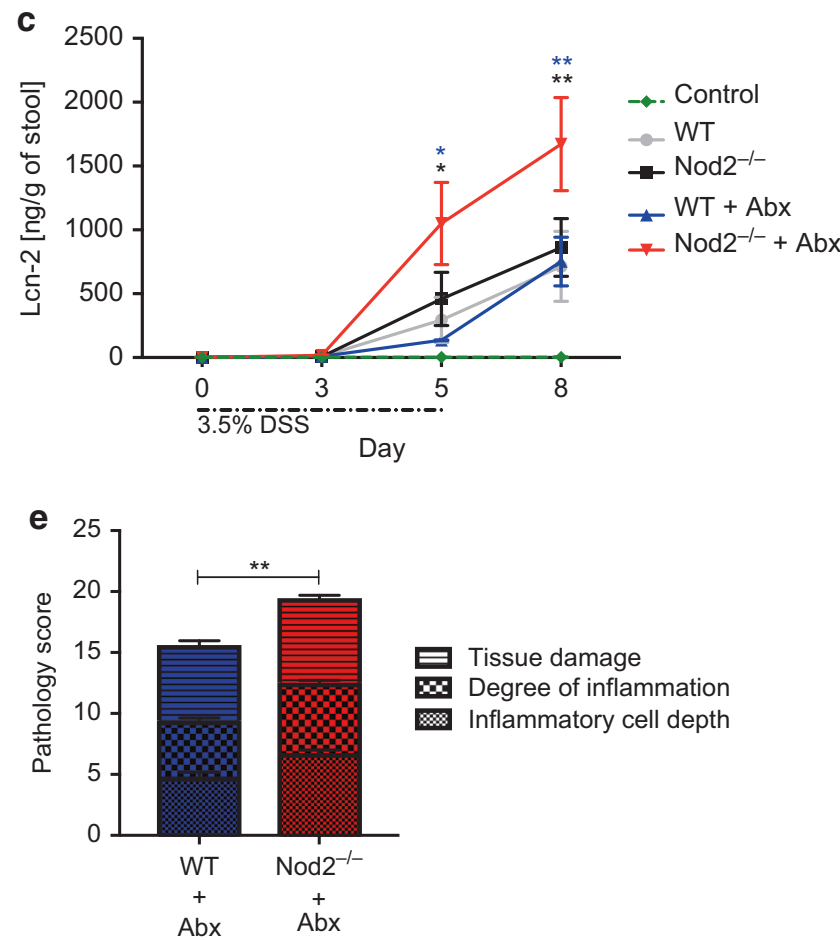
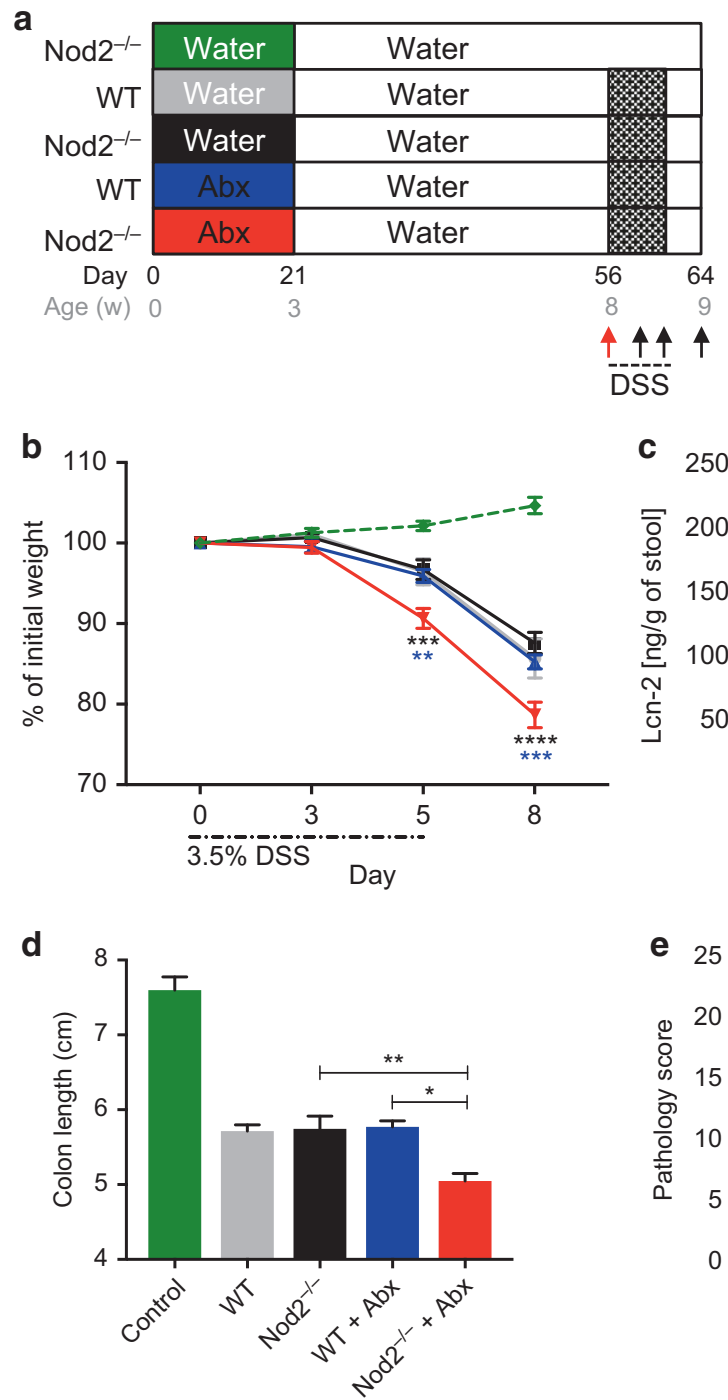

a

$\mathbf{f}$
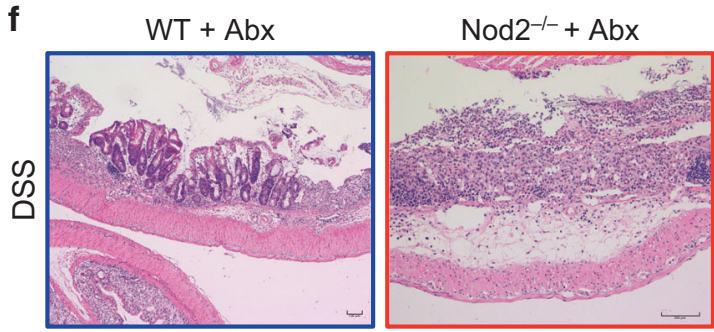

Fig. 4 Neonatal antibiotic treatment increases susceptibility to DSS colitis in Nod2 ${ }^{-/-}$mice 5 weeks later. a Experimental timeline. Mice were given antibiotic-supplemented water or untreated water following birth until weaning on day 21. Colitis was induced on day 56 (when mice were 8 weeks of age) by providing 3.5\% DSS water for 5 days. Tissues were analyzed on day 8 from DSS induction. Untreated Nod2 ${ }^{-1-}$ mice that did not receive DSS served as negative controls (green). b Percent of initial weight over time. c Fecal lipocalin-2 concentration was measured by ELISA and normalized to stool weight. $\mathbf{d}$ Colon length, measured on day 8. e Colonic pathology score on day 8 . $\mathbf{f}$ Representative photographs at $\times 100$ magnification of H\&E stained sections of the colon on day 8 . Data are presented as mean \pm SEM. ${ }^{*} p<0.05,{ }^{* *} p<0.01$, ${ }^{* * *} p<0.001,{ }^{* * * *} p<0.0001$; by two-way ANOVA and post hoc Holm-Šídák test $(\mathbf{b}, \mathbf{c})$ and Mann-Whitney $U$ test $(\mathbf{d}, \mathbf{e})$. ( $n=9-19$ mice per genotype, per treatment from five untreated litters and six antibiotic-treated litters) 
Enterobacteriaceae, along with diminished abundance of Akkermansia, Parabacteroides, and Adlercreutzia (Supplementary Figure 1C). Together, these data suggest a role for Nod2 in shaping the microbial changes at weaning in response to neonatal antibiotic exposure.

LEfSe analysis of the microbiota on day 35 further highlighted the differential response to and recovery from antibiotic treatment in Nod2-deficient mice (Fig. 3b), including increased Prevotellaceae and Lactobacillaceae, and decreased Clostridaceae, compared to water-treated controls. Comparison between antibiotictreated WT and $\mathrm{Nod}^{-/-}$littermates emphasized the loss of Erysipelotrichaceae and over-abundance of Coriobacteriaceae in the $\mathrm{Nod}^{-/-}$mice as the strongest differences between the genotypes (Supplementary Figure 1D). Clustering analysis further supported these findings (Fig. 3c). Together, Nod2-deficiency was associated with residual changes in the microbiota and a failure to fully recover to the composition and diversity of water-treated mice. These data suggest that Nod2 not only influences the specific response of the microbiota to antibiotic treatment, but also influences the recovery, suggesting that microbial changes may persist long after the antibiotic perturbation.

Adult antibiotic exposure fails to increase susceptibility to DSS colitis

Antibiotic exposure has been associated with IBD development, however the mechanism remains unclear. Given that a seven-day course of antibiotics had long-lasting impact on the gut microbiota of adult Nod2 $2^{-1-}$ mice, we assessed whether this perturbed microbiota altered susceptibility to or severity of experimental colitis. Colitis was induced in adult mice 4 weeks after completion of antibiotic treatment (Supplementary Figure 2A). Water and antibiotic-treated WT and Nod2 ${ }^{-1-}$ littermates were subjected to DSS-supplemented drinking water for 5 days. Individual body weight was recorded and the level of lipocalin-2
(Lcn-2) (an anti-microbial peptide produced by neutrophils and epithelial cells and released during intestinal inflammation ${ }^{17}$ ) in stool was measured on day 0,3 , and 5 of DSS treatment. DSS exposure resulted in significant weight loss, which was not altered by Nod2 genotype or antibiotic exposure (Supplementary Figure 2B). Lipocalin-2 was also significantly increased in DSStreated mice, but no differences were identified between genotypes or antibiotic treatment (Supplementary Figure 2C). Colon length and pathological colitis score further supported no effect of antibiotic exposure on severity of DSS colitis in WT or Nod2 ${ }^{-\prime-}$ littermate mice (Supplementary Figure 2D-F). These data suggest that although microbiota of adult $\mathrm{Nod} 2^{-1-}$ mice had delayed resilience following antibiotic treatment, there was no effect on susceptibility or severity to DSS-induced mucosal damage.

Neonatal antibiotic treatment increases susceptibility to DSS colitis 35 days after antibiotics in Nod2 $2^{-1-}$ mice

We next assessed whether the neonatal antibiotic treatment had an effect on susceptibility to DSS-induced colitis. As described above, at weaning mice were placed on normal drinking water for 5 weeks, and colitis was initiated on day 56 (Fig. 4a). Starting on day 56 (day 0 of disease induction), mice were fed DSSsupplemented drinking water for 5 days followed by a 2-day rest on regular drinking water and tissues were analyzed on day 64. DSS caused water-treated WT and Nod2 $2^{-1-}$ mice and antibiotictreated WT mice to lose $\sim 10 \%$ of their initial body weight by day 8 , whereas antibiotic-treated $\mathrm{Nod}^{-/-}$mice lost over $20 \%$ of starting weight (Fig. 4b). Stool collected on days $0,3,5$, and 8 was used to quantify fecal lipocalin-2 concentration. Corresponding with the increased weight loss, antibiotic-treated $\operatorname{Nod}^{-1-}$ had elevated lipocalin-2 concentration at days 5 and 8 reflecting more significant inflammation (Fig. 4c). Furthermore, antibiotic-treated Nod $2^{-1-}$ mice had greater colonic shortening compared to a

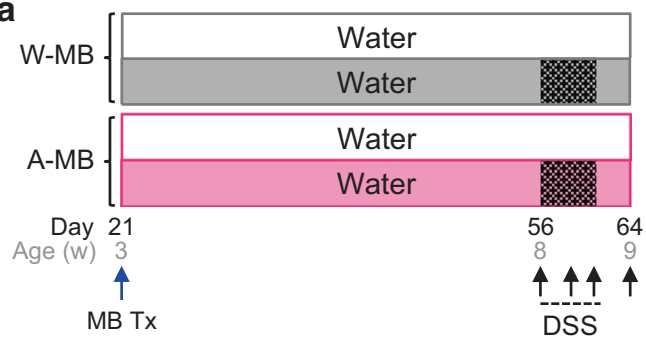

b

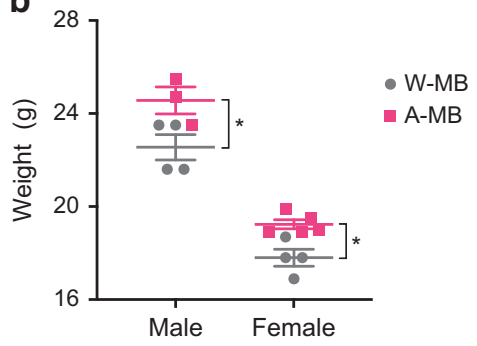

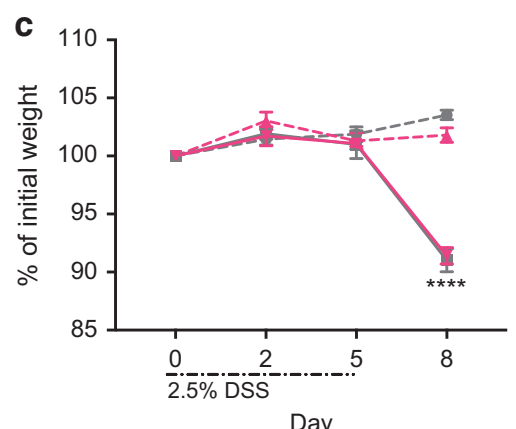

Day
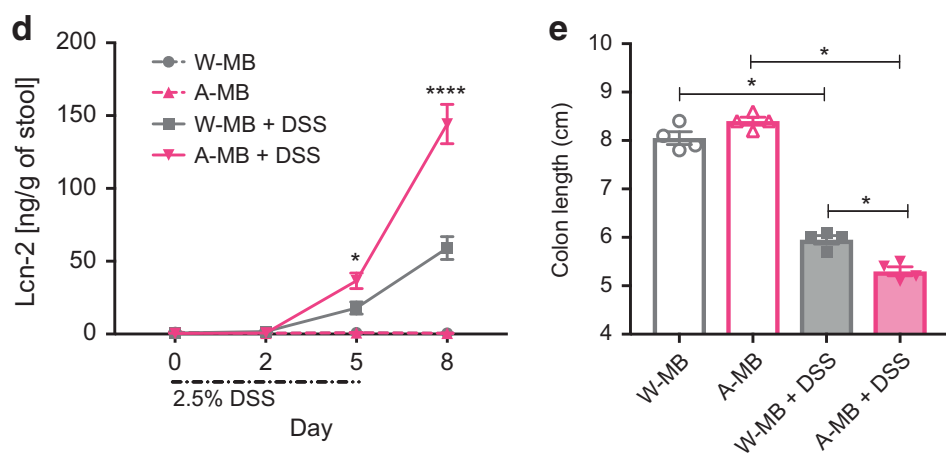

Fig. 5 Microbiota transferred from antibiotic-treated neonatal SPF mice to germ-free Nod2-1- mice increases DSS colitis severity. Watertreated (W-MB) or antibiotic-treated (A-MB) microbiota from the cecum was collected from SPF Nod2 ${ }^{-1-}$ mice at 21 days of age, and transplanted into age-matched germ-free $\mathrm{Nod}^{-1-}$ mice. Recipient mice were left alone for 5 weeks and then challenged with DSS colitis. a Experimental timeline. Germ-free Nod2 $2^{-1-}$ mice received either water-treated (W-MB) or antibiotic-treated (A-MB) microbiota at weaning (day 21). At 8 weeks old (day 56), DSS colitis was induced using $2.5 \% \mathrm{w} / \mathrm{v}$ DSS in non-acidified drinking water. $\mathbf{b}$ Weight of male and female W$M B$ and A-MB recipients on day 56. c Percent of initial weight over time. d Fecal lipocalin-2 concentration was measured by ELISA and normalized to stool weight. e Colon length, measured on day 8. Data are presented as mean \pm SEM. ${ }^{*} p<0.05,{ }^{* * * *} p<0.0001$; by two-way ANOVA and post hoc Holm-Š́lák test (b-d), and Mann-Whitney $U$ test (e). (W-MB $n=4, A-M B ~ n=4, W-M B+D S S n=4, A-M B+D S S n=4)$ 

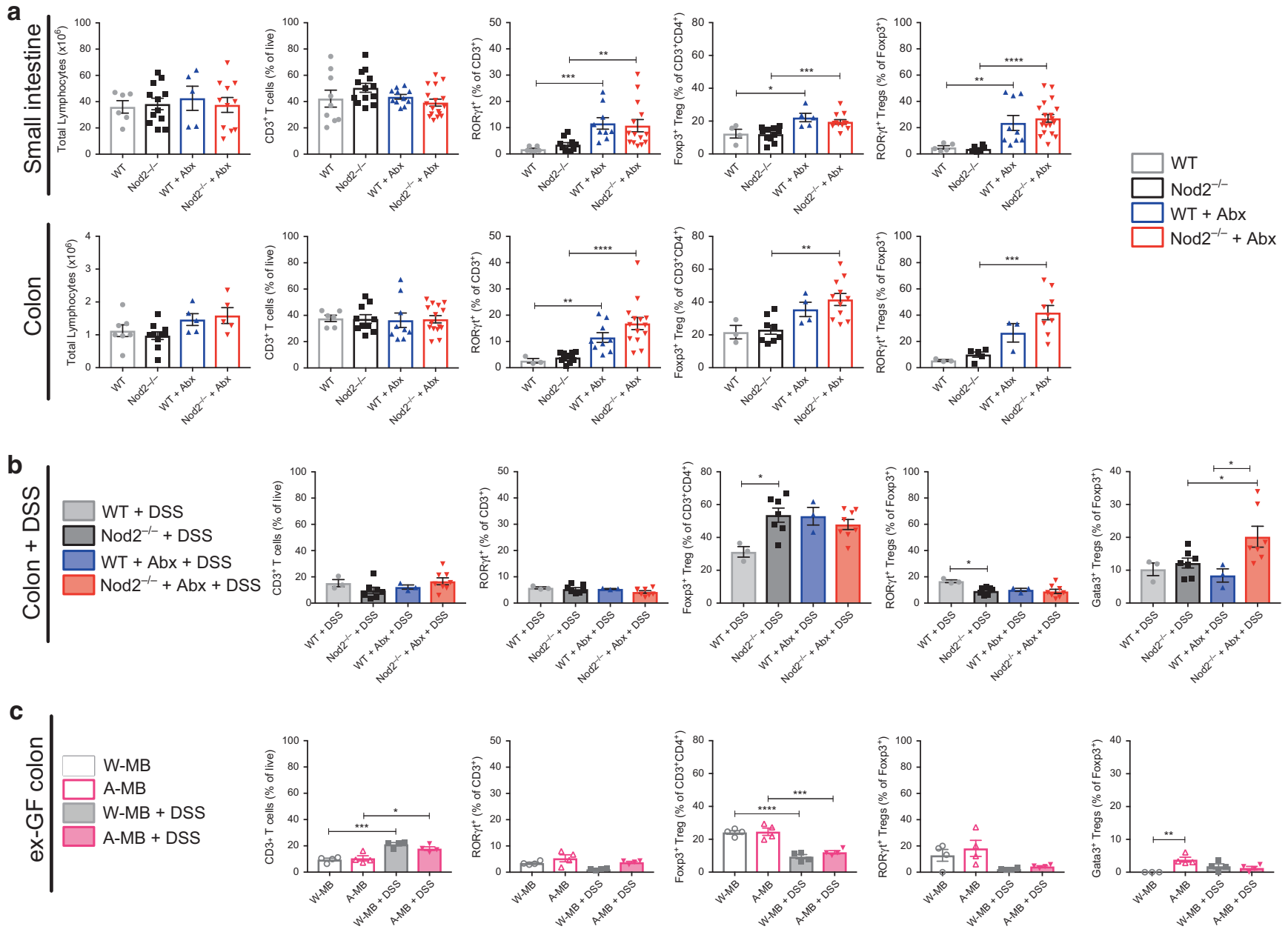

Fig. 6 Neonatal antibiotic treatment alters microbially driven T cell populations in the gut. a T cell profile of the small intestinal and colonic lamina propria from water and antibiotic-treated WT and Nod $2^{-1-}$ littermates at 8 weeks of age. a Number of total lymphocytes, and percent of $\mathrm{CD}^{+}{ }^{+}, \mathrm{ROR}_{\mathrm{t}} \mathrm{t}^{+}, \mathrm{Foxp}^{+}$, and ROR $\mathrm{t}^{+}$Foxp $3^{+}$double positive T cells within the intestinal lamina propria. $(n=6-13$ mice per genotype, per treatment from four untreated and four antibiotic-treated litters). b Colonic T cell profile from water and antibiotic-treated WT and Nod $2^{-1-}$ littermates after DSS. Percent of $\mathrm{CD}^{+}{ }^{+}, \mathrm{ROR}_{\mathrm{t}}{ }^{+}$, Foxp $3^{+}, \mathrm{ROR}_{\mathrm{t}}{ }^{+} \mathrm{Foxp}^{+}$, and $\mathrm{Gata}^{+} \mathrm{Foxp}^{+}$double positive $\mathrm{T}$ cells within the colonic lamina propria. (WT+DSS $n=3$, Nod2 $2^{-1-}+\mathrm{DSS} n=7$, WT+Abx+DSS $n=3$, Nod2 ${ }^{-1-}+$ Abx+DSS $n=8$ ). c Colonic T cell profile from ex-germ-free W-

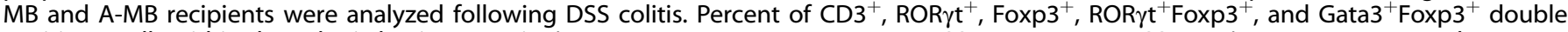
positive T cells within the colonic lamina propria. (W-MB $n=4, \mathrm{~A}-\mathrm{MB} n=4, \mathrm{~W}-\mathrm{MB}+\mathrm{DSS} n=4, \mathrm{~A}-\mathrm{MB}+\mathrm{DSS} n=4$ ). Data are presented as mean \pm SEM. ${ }^{*} p<0.05,{ }^{* *} p<0.01,{ }^{* *} p<0.001,{ }^{* * *} p<0.0001$; by Mann-Whitney $U$ test $(\mathbf{a}, \mathbf{b})$, and one-way ANOVA and post hoc Tukey test $(\mathbf{c})$

water-treated Nod $2^{-1-}$ mice and antibiotic-treated WT littermates (Fig. 4d). Pathological scoring also indicated increased tissuedamage and inflammatory cell depth in antibiotic-treated Nod $2^{-1-}$ mice compared to WT littermates (Fig. 4e, f). No differences in colitis susceptibility or severity were observed between water-treated and antibiotic-treated WT mice, nor were differences observed between water-treated WT and Nod2deficient littermates, indicating that the antibiotic perturbation in early life, combined with a Nod2-deficiency, resulted in worsened disease.

Next, we sought to determine whether the microbiota was having a causal role in the increased colitis severity. Cecal microbiota was collected at weaning from water or antibiotictreated $\mathrm{Nod}_{2}{ }^{-1-}$ mice and transplanted into age-matched germfree $N o d 2^{-1-}$ recipients. Colitis was induced in the recipients at 8 weeks of age (day 56) (Fig. 5a). Both male and female recipients of the antibiotic-treated microbiota $(A-M B)$ weighed more than their counterparts who received water-treated microbiota (W-MB) on day 56 (Fig. 5b), supporting previous studies that showed an effect of antibiotic exposure on weight gain. ${ }^{18}$ Although both A-MB and $\mathrm{W}-\mathrm{MB}$ recipients lost $\sim 10 \%$ body weight following DSS exposure (Fig. 5c), A-MB recipients had significantly increased fecal lipocalin-2 on day 5 and 8 and shorter colons indicating more severe damage (Fig. $5 \mathrm{~d}$, e). Together, this demonstrates that the antibiotic-altered microbiota was sufficient for the development of a more severe DSS-induced colitis in the germ-free $\mathrm{Nod}^{-1-}$ recipients.

Colonic regulatory $\mathrm{T}$ cells are induced by altered gut microbiota and regulate colitis

Regulatory T cells $\left(\mathrm{T}_{\text {reg }}\right.$ ) are involved in controlling and limiting gut inflammation and can be induced through microbial interactions. ${ }^{19-23}$ We postulated that differences in $\mathrm{T}_{\text {reg }}$ could be involved in the increased susceptibility to DSS observed in the neonatal antibiotic-treated $\mathrm{Nod}^{-1-}$ mice and the A-MB gnotobiotic recipients. In order to address this, we performed flow cytometry on small intestinal and colonic lamina propria lymphocytes from neonatal water-treated and antibiotic-treated WT and Nod2 ${ }^{-1-}$ littermates at 8 weeks of age. There were no differences in total lymphocytes isolated or in the percent of $\mathrm{CD}^{+} \mathrm{T}$ cells between genotypes and treatments (Fig. 6a). RORyt ${ }^{+} \mathrm{T}$ cells, Foxp3 ${ }^{+}$ regulatory $\mathrm{T}$ cells, and $\mathrm{ROR}_{\mathrm{ft}} \mathrm{F}^{+} \mathrm{Foxp}^{+}$double positive $\mathrm{T}_{\text {reg }}$ were 

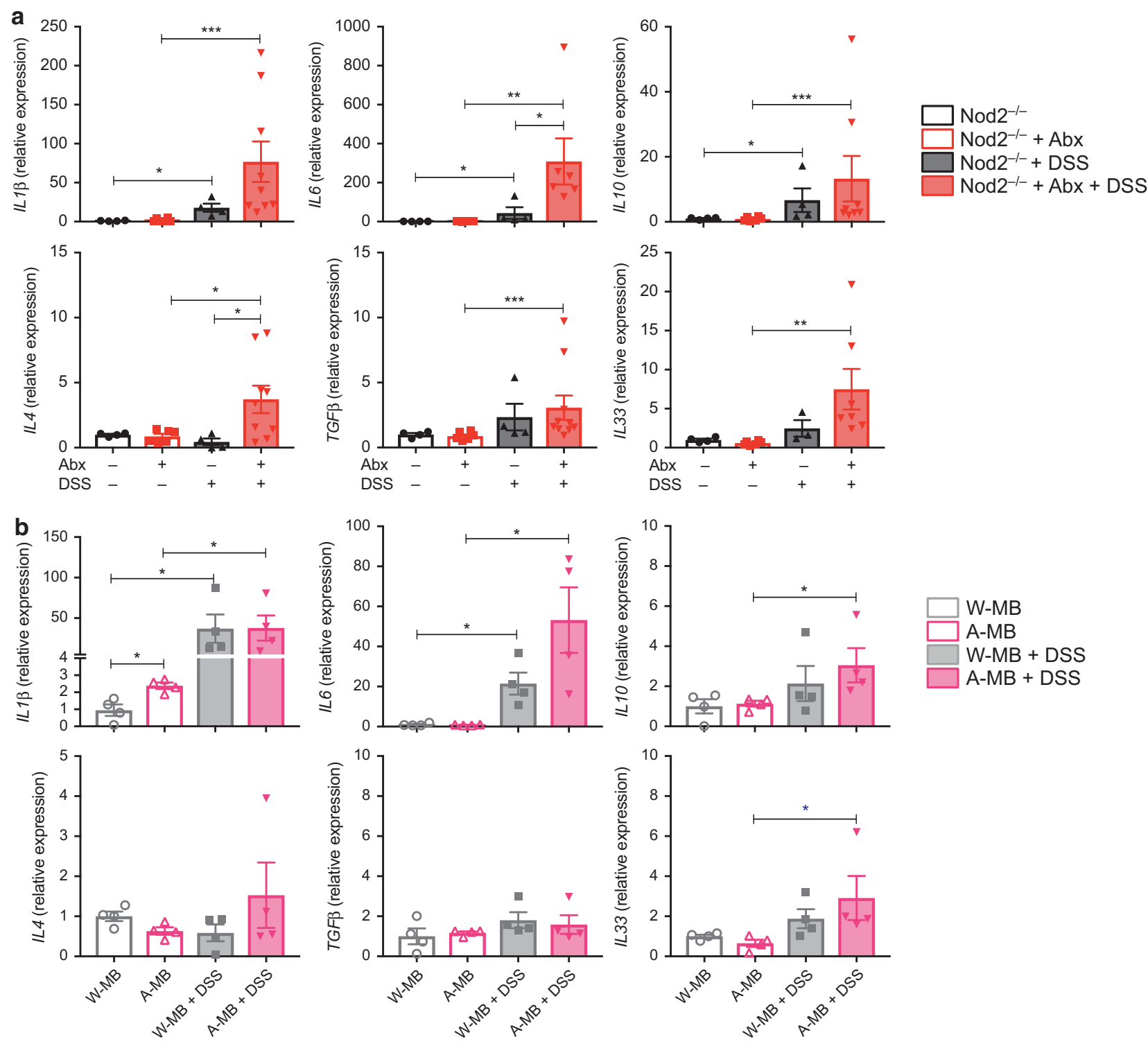

Fig. 7 DSS exposure alters the cytokine milieu in the colon of neonatal antibiotic-treated Nod $2^{-1-}$ mice. a Cytokine expression in the proximal colon of water-treated and antibiotic-treated $\mathrm{Nod}_{2}^{-1-}$ mice was assessed by quantitative PCR. b Cytokine expression in the proximal colon of $\mathrm{W}-\mathrm{MB}$ and $\mathrm{A}-\mathrm{MB} \mathrm{Nod2}{ }^{-1-}$ recipient mice was assessed by quantitative PCR. Data are presented as mean \pm SEM. ${ }^{*} p<0.05,{ }^{* *} p<0.01,{ }^{* * *} p<$ 0.001 ; by Mann-Whitney $U$ test. ( $n=4-10$ mice per genotype, per treatment)

significantly increased in the small intestine of mice previously treated with antibiotics compared to water controls (Fig. 6a). Interestingly, although the RORyt ${ }^{+} \mathrm{T}$ cell populations were similar in the small intestine and colon, the $T_{\text {reg }}$ increases were only seen in the colonic lamina propria of Nod2-deficient littermates, although there was a trend for increases in the antibiotictreated WT littermates. These T cell populations are known to be induced via host-microbe interactions. ${ }^{24}$

We next explored the effect of DSS-induced damage on colonic T cell populations. DSS exposure induced an increase in Foxp3 ${ }^{+}$ $\mathrm{T}_{\text {reg }}$ in the colon of water-treated $\mathrm{Nod}^{-1-}$ mice but did not further increase the already elevated population in antibiotictreated mice. RORyt ${ }^{+}$Foxp $^{+}$double positive $\mathrm{T}_{\text {reg }}$ were significantly diminished in the DSS-exposed, antibiotic-treated mice, and in water-treated $\mathrm{Nod}^{-/-}$mice compared to WT littermates (Fig. 6b). Coinciding with the reduction in RORyt ${ }^{+}$Foxp $^{+}$double positive $T_{\text {reg, }}$ we detected a significant increase in Gata3expressing Foxp3 ${ }^{+} \mathrm{T}_{\text {reg }}$ in antibiotic-treated $\mathrm{Nod}^{-1-}$ mice following DSS exposure that was not observed in antibiotictreated WT mice or water-treated controls (Fig. 6b).

Analysis of the colonic lymphocytes from $W-M B$ and $A-M B$ Nod $2^{-1-}$ recipients showed no differences in the proportions of $\mathrm{CD}^{+}$, RORyt $^{+}$, Foxp3 $^{+}$, or RORyt ${ }^{+}$Foxp3 $^{+}$T cells between A-MB and W-MB recipients (Fig. 6c, open bars). However, although W-AB recipients had no Gata3 ${ }^{+} \mathrm{Foxp}^{+} \mathrm{T}_{\text {reg, }}$ A-MB recipients had $\sim 4 \%$ of $\mathrm{T}_{\text {reg }}$ co-expressing Gata3 ${ }^{+}$and Foxp ${ }^{+}$. DSS-induced colitis caused an increase in the proportion of $\mathrm{CD}^{+}{ }^{+}$cells, but reduced RORpt ${ }^{+}$, Foxp $^{+}$, and RORYt ${ }^{+}$Foxp $^{+}{ }^{+}$T cells in both $\mathrm{W}-\mathrm{MB}$ and $\mathrm{A}-\mathrm{MB}$ recipients (Fig. $6 c$, shaded bars). Although the reduction of RORyt ${ }^{+}$Foxp $3^{+} \mathrm{T}_{\text {reg }}$ in the gnotobiotic recipients is similar to what was observed in the SPF mice, there was no coinciding expansion of $\mathrm{Gata}^{+}{ }^{+} \mathrm{Foxp}^{+} \mathrm{T}_{\text {reg }}$ after DSS. Thus, while transfer of antibiotictreated microbiota was sufficient to recapitulate the increased colitis phenotype observed in Nod2 ${ }^{-1-}$ mice, the effect on mucosal $T_{\text {reg }}$ was not the same, possibly due to the use of the germ-free colonization model. Indeed, it is difficult to ascertain whether the differences were due to the colonization timing, or 
were secondary phenomena following DSS-induced mucosal damage.

DSS exposure alters the colonic cytokine milieu of $\mathrm{Nod}^{-1-}$ mice with neonatal antibiotic-treated microbiota

In order to examine the cytokines involved in the DSS response and possibly influencing the change in $T_{\text {reg }}$ response, mRNA from the proximal colon of water and antibiotic-treated $\mathrm{Nod}^{-1-}$, as well as $\mathrm{W}-\mathrm{MB}$ and $\mathrm{A}-\mathrm{MB}$ recipient Nod2 $2^{-1-}$ mice, was analyzed. DSS-induced colitis resulted in increased expression of the proinflammatory cytokines ILI $\beta$ and IL6 in both water and antibiotictreated $\mathrm{Nod} 2^{-1-}$ mice, with a further increase in IL6 expression in antibiotic-treated mice (Fig. 7a), complementary to the increased weight loss and pathology observed. Expression of the antiinflammatory cytokine IL10 was also induced with DSS exposure, but no difference was detected between water and antibiotictreated mice (Fig. 7a). Interestingly, A-MB recipients had increased expression of $I L 1 \beta$ compared to $\mathrm{W}-\mathrm{MB}$ recipients in the absence of inflammation; however, the intense damage induced by DSS resulted in increased ILI $\beta$ and IL6 in both $\mathrm{W}-\mathrm{MB}$ and $A-M B$ recipients (Fig. 7b). Expression of ILIO was only significantly increased in A-MB recipients.

Gata3 expression in Foxp3 ${ }^{+} \mathrm{T}_{\text {reg }}$ can be induced through the cytokines IL-4, TGF $\beta$ and IL-33. Indeed, the proportion of Foxp3 ${ }^{+}$ $\mathrm{T}_{\text {reg }}$ expressing Gata3 was significantly increased in antibiotictreated Nod2 $2^{-1}$ mice exposed to DSS and A-MB recipients (Fig. $6 \mathrm{~b}, \mathrm{c}$ ). Expression of both $I L 10$ and TGF $\beta$ contribute to $\mathrm{T}_{\text {reg }}$ maintenance, and enhanced IL4 and IL33 expression suggests that the cytokine milieu favored the generation and support of Gata $^{+} \mathrm{Foxp}^{+} \mathrm{T}_{\text {reg }}$ during DSS colitis in antibiotic-treated Nod $2^{-1-}$ mice (Fig. 7a). Although no differences in IL4 and TGF $\beta$ expression were detected in $\mathrm{W}-\mathrm{MB}$ or $\mathrm{A}-\mathrm{MB}$ recipients, IL33 expression was significantly increased in A-MB mice after DSS (Fig. 7b). Thus, the cytokine milieu is influenced by both the gut microbiota and DSS-induced inflammation. Coinciding with the macroscopic and $\mathrm{T}$ cell changes observed, DSS colitis combined with an antibiotic-altered microbiota resulted in increased expression of pro-inflammatory cytokines and favored the expansion of a unique population of $\mathrm{Gata}^{+}{ }^{+}$ Foxp $^{+} \mathrm{T}_{\text {reg. }}$.

Together, our data show that neonatal antibiotic treatment can alter the gut microbiota long-term and influence colitis susceptibility in a Nod2-deficient host. Although Nod2 alters the response of the gut microbiota to antibiotics in adult mice, this fails to increase colitis susceptibility, suggesting that the timing of the antibiotic exposure is key to the influence of the microbial changes on the mucosal immune response.

\section{DISCUSSION}

In this study, we investigated the effect of antibiotic treatment on the composition and diversity of the gut microbiota and whether Nod2, the strongest IBD genetic risk association and a microbial sensor, influenced the response. We found that Nod2-deficient mice had prolonged microbial perturbation following antibiotic treatment, both as neonates and adults. Although adult antibiotic treatment had no effect on DSS colitis, Nod2 ${ }^{-1-}$ mice that were exposed as neonates were more susceptible to colitis, with increased weight loss and mucosal damage. Colonization of germfree $\mathrm{Nod} 2^{-1-}$ mice with neonatal antibiotic-treated microbiota resulted in worsened colitis compared to recipients of watertreated microbiota. Together, these data illustrate the broad and long-lasting effects of antibiotic perturbation of the microbiota in early life, and how host genetics can alter these effects and lead to increased susceptibility to colitis. Moreover, these data indicate that Nod2 has a critical role in shaping gut microbial responses and resilience to perturbations.
Antibiotic exposure transiently changes the gut microbiota in humans; ${ }^{25,26}$ however, the microbiota is usually resilient, returning to pre-treatment composition shortly after removal of the antibiotic for most subjects. ${ }^{25,27}$ Alterations in the microbiota of both antibiotic-treated neonatal and adult Nod2 $2^{-1-}$ mice were maintained 14 days after the removal of antibiotic treatment suggesting a reduced resilience. Our study is the first to identify a role for Nod2 in microbial resilience following an antibiotic perturbation.

Neonatal antibiotic exposure has been associated with several auto-inflammatory diseases including asthma, obesity, and IBD. ${ }^{8,9,11}$ In humans, the microbiota is highly malleable until 2-3 years of age. ${ }^{3,28}$ In order to model the effects of repeated antibiotic exposure during the first years of an infant's life (but not influence the original inoculum that birth provides), we developed an antibiotic treatment regime, starting after birth until weaning, using the most commonly prescribed antibiotic to pediatric patients, amoxicillin. ${ }^{29}$ The broad-spectrum beta-lactam, effective against Gram- negative and positive bacteria, also passes through breast milk, making it an ideal candidate for antibiotic exposure to pups that avoids the stress and potential concomitant alterations of the microbiome due to handling. ${ }^{30,31}$ We found that amoxicillin treatment caused a significant shift in the community structure of the gut microbiota in mice at weaning, with reduced bacterial richness and diversity. Antibiotic treatment resulted in loss of Bifidobacteriaceae, Lactobacillaceae, Lachnospiraceae, and Erysipelotrichaceae, whereas Bacteroidaceae and Enterobacteriaceae bloomed. These changes are in agreement with antibiotic-induced alterations observed previously in both humans and mice. ${ }^{18,28,32,33}$ Specifically, the maintained loss of Lachnospiraceae and expansion of Porphyromonadaceae following antibiotic treatment was also observed in a longitudinal study in children followed from birth until 2 years of age. ${ }^{32}$

Corroborating previous work by our team, there was no effect of Nod2 genotype on the stool microbial composition between water-treated littermates. ${ }^{14}$ However, we identified several differences in the microbiota between antibiotic-treated WT and Nod2 $2^{-1-}$ littermates, in both adult and neonates. Antibiotictreated $\mathrm{Nod}^{-/-}$mice displayed a persistent loss of Erysipelotrichaceae and increased levels of Prevotellaceae two weeks after removal of the antibiotic treatment. The idea of a 'pro-colitogenic' microbiota in Nod2-deficient mice has been described previously; ${ }^{34-36}$ however, those studies used non-littermate mice and so the exact influence of Nod2 on the microbiota and colitis susceptibility is not clear. We used littermate mice in order to assess the specific influence of Nod2 genotype on the gut microbiota and responses to antibiotic perturbation, while controlling and normalizing other environmental influences. ${ }^{37}$ The microbiota transfer experiment highlights the influence of Nod2 genotype on the microbiota, since Nod2-deficient recipients of the A-MB were more susceptible to colitis; however, it remains to be shown whether this is due to the influence of Nod2 genotype on the donor microbiota versus on the recipient's response to the altered microbes. Although direct comparisons to human data cannot be made, this study emphasizes the role of host genetics on shaping the gut microbiota in response to environmental perturbations.

We examined the effects of prior antibiotic exposure on colitis susceptibility and severity. First, the DSS model was utilized to induce colitis in mice that received antibiotic treatment as adults, however no effect of genotype or treatment was observed on susceptibility or severity. This is consistent with previous findings showing that Nod2-sufficient and deficient littermates do not differ in response to DSS, ${ }^{38}$ but contradicts other papers where non-littermate mice (with different gut microbiota) showed increased susceptibility of Nod2 $2^{-1-}$ to DSS-induced colitis. ${ }^{34,39}$ On the other hand, neonatal antibiotic treatment of $\mathrm{Nod}^{-1-}$ littermates resulted in worsened colitis, characterized by increased 
weight loss and mucosal damage. The difference in colitis susceptibility between the adult and neonatal antibiotic treatment within the same littermate colony indicates that the time of the exposure is critical for how the immune system responds to microbial perturbations. Some studies have successfully described the effects of different classes of antibiotics on the gut microbiota in children, ${ }^{28,40}$ and others have gone on to implicate these changes with disease development later in life. ${ }^{41}$ Recently, two groups reported similar studies assessing the effects of peripartum antibiotic exposure or transfer of antibiotic-altered microbiota in the $I L-10^{-1-}$ model of colitis. ${ }^{12,42}$ These two studies also observed that antibiotic-altered gut microbes resulted in increased susceptibility to colitis. Although the $I L-10^{-1-}$ model is an excellent mouse model for assessing microbially driven inflammation (as mice do not develop disease when maintained germ-free, ${ }^{43}$ ) mutations in the IL-10 pathway results in severe immune dysregulation and very early onset-IBD (VEO-IBD) in humans. ${ }^{44}$ Since NOD2 mutations have the strongest genetic-risk association with IBD and as a cytosolic microbial receptor, our study provides a unique look at the influence of IBD-related host genetics on the response to antibiotic exposure, the resilience of the microbiota and susceptibility to colitis.

Antibiotic treatment increased intestinal $T$ cell populations involved in host-microbe interactions, including $T_{\text {reg, }}$ Th17, and RORyt ${ }^{+}$Foxp $^{+} \mathrm{T}_{\text {reg. }}$ In contrast to a study that found that neonatal vancomycin treatment reduced colonic Foxp3 ${ }^{+} \mathrm{T}_{\text {reg, }}{ }^{45}$ our neonatal amoxicillin treatment increased intestinal Foxp3 ${ }^{+} \mathrm{T}_{\text {reg }}$ populations. Our findings point to the idea that the development of intestinal $T_{\text {reg }}$ may be complex and tightly linked to the gut microbiota. Induction of non-classical $\mathrm{T}_{\text {reg, }}$ including RORyt ${ }^{+}$Foxp $^{+}$and Gata3 $^{+}$Foxp3 $^{+} \mathrm{T}_{\text {reg, }}$ in our antibiotic-treated mice indicates the important influence of microbes on the maturation of these cells. Indeed, previous studies have suggested that these cells are generated in response to the gut microbiota. ${ }^{46,47}$ RORyt $^{+}$Foxp3 $^{+} \mathrm{T}_{\text {reg }}$ develop from Foxp3 ${ }^{+} \mathrm{T}_{\text {reg, }}{ }^{48}$ and are functionally equivalent, if not superior, to Foxp3 $3^{+} \mathrm{T}_{\text {reg }}$ in protecting against inflammation in the $T$ cell transfer model of colitis. ${ }^{49}$ By contrast, Gata3 ${ }^{+}$Foxp $^{+} \mathrm{T}_{\text {reg }}$ are driven by IL-33 released during barrier disruption, mediate tissue damage, ${ }^{50}$ and are thought to be involved with controlling inflammation and mucosal damage. ${ }^{51,52}$ Previously, these cells were shown to not differ in abundance between germ-free and SPF WT mice; ${ }^{53}$ however, whereas the A-MB Nod2 $2^{-1-}$ recipients had colonic $\mathrm{Gata}^{+}{ }^{+} \mathrm{Foxp}^{+} \mathrm{T}_{\text {reg, }}$, the W-MB recipients had none. This difference in findings may indicate that these cells can be microbially induced. Together, this suggests that the altered intestinal $T$ cell populations following neonatal antibiotic treatment can result in dysregulated immune responses and worsened colitis in a genetically susceptible host.

Antibiotic exposure is associated with increased risk for IBD development, however the differences in the gut microbiota between individuals make it difficult to ascertain the causal effect of antibiotic exposure. Using a controlled animal model, we identified that Nod2-deficiency resulted in delayed resilience of the gut microbiota following adult and neonatal antibiotic treatment; however, only Nod2 $2^{-1-}$ mice exposed as neonates experienced an increased severity of colitis indicating that time of exposure is critical. Collectively, our data highlight the long-lasting influence of an altered neonatal microbiota on mucosal immune homeostasis and development of disease.

\section{MATERIALS AND METHODS}

Mice

Wild-type (WT), Nod2 ${ }^{-/-}$, and Nod2 ${ }^{+/-}$littermates (on a C57BL/6 background) were bred in-house under specific pathogen-free conditions. Littermate breeders were maintained in heterozygous $(\mathrm{Het}) \times$ Het crosses, using sister dams, whenever possible. Mice received breeder chow (Teklad $^{\circledR}$ irradiated Rodent Chow \#2919; Envigo, Huntingdon, UK) and autoclaved drinking water ( $\mathrm{pH} \sim 7$ ) ad libitum. All mice were housed on the same rack within the facility and received normal chow (Teklad $^{\circledR}$ irradiated Rodent Chow \#2918; Envigo) and autoclaved drinking water (pH $~ 7)$ ad libitum from weaning to endpoint.

Germ-free Nod2-deficient mice were kindly provided by Drs. Jon Schertzer and Elena Verdu from McMaster University, Hamilton, Ontario, Canada.

All experiments were performed in accordance with University of Toronto's Department of Comparative Medicine approved animal use protocols.

\section{Antibiotic treatment}

Antibiotic (Abx)-treated mice received amoxicillin (BioShop, Burlington, Canada) at $200 \mathrm{mg} / \mathrm{L}$ in autoclaved drinking water ( $\mathrm{pH} \sim 7)$. Adult treatment started at 8 weeks of age and was maintained for 7 days (Figs. 1a and 2a). Neonatal treatment started one day after birth and was maintained until weaning (day 21), followed by autoclaved drinking water from weaning to endpoint (Fig. 3a). Bottles were refreshed every other day. Water-treated mice received autoclaved drinking water for the duration of all experiments.

\section{$16 \mathrm{~S}$ sequencing and analysis}

The V4 hypervariable region of bacterial 16S ribosomal DNA (16S rDNA) was sequenced in paired-end modus $(2 \times 150$ base pair) on the MiSeq platform (Illumina, San Diego, CA) using primers 515F/ $806 \mathrm{R}^{54}$ The resulting paired reads were assembled using PANDAseq $v 2.7$ to generate an amplicon size of 250 base pairs. ${ }^{55}$ Sequences were de-multiplexed and processed by the quantitative insights into microbial ecology (QIIME v1.8.0) pipeline using default parameters. ${ }^{56}$ The mean number of quality-controlled reads was $72,760 \pm 33,893$ (mean $\pm S D$ ) per mouse sample. Chimeric sequences were identified de novo, reference-based and then removed using usearch61. ${ }^{57}$ The non-chimeric sequences were then clustered into operational taxonomic units (OTUs) at $97.0 \%$ sequence similarity using a closed referencebased picking approach with UCLUST software against Greengenes database 13_8 of bacterial $16 \mathrm{~S}$ rRNA sequences. ${ }^{58}$ After rarefaction at 10,000 reads per sample, bacterial alpha diversity was estimated using the Chao 1 and Shannon diversity indices. Samples with fewer than 10,000 reads after quality filtering were removed from the analysis. OTUs with a prevalence $<5 \%$ were removed from the analysis. Analyses using R software v2.14.1 were restricted to merged OTUs with the same taxonomic assignment. A paired Student's $t$-test was applied to compare the alpha diversity differences in microbial profiles in WT and Nod2 ${ }^{-1-}$ mice, and between antibiotic and untreated groups. Relative abundance of taxa in microbial profiles in WT and $\mathrm{Nod}^{-1-}$ mice was compared using the Kruskal-Wallis test. Associations between bacterial taxa and groups were considered to be significant after a false-discovery rate (FDR) correction of the $p$-value $(q<0.05)$. Beta diversity was assessed using Bray-Curtis dissimilarity distances. Procrustes analyses based on Bray-Curtis distance was applied to compare microbial orientation differences following antibiotic treatment and a Monte Carlo simulation was performed using 10,000 permutations on the first five dimensions of the PCoA. Microbial community similarity between the different groups of mice was addressed by performing an ANOSIM test with 10,000 permutations on the beta-diversity metrics described above on the first five dimensions of the PCoA.

Microbiota transfer

Cecal contents from water-treated or antibiotic-treated Nod2 $2^{-1-}$ littermates were harvested anaerobically at weaning and diluted $1 / 10$ in $50 \%$ glycerol. Germ-free C57BL/6 Nod2 $2^{-1-}$ mice were bred in-house at the University of Toronto Gnotobiotic facility. At 
weaning, under strict aseptic conditions, germ-free mice were transferred into the specific pathogen-free facility and colonized with $150 \mu \mathrm{L}$ of the cecal content slurry. Colitis was induced 5 weeks post-colonization.

Statistical analysis

Results are depicted as mean \pm SEM, unless otherwise indicated. Statistical analysis was performed using one-way or two-way analysis of variance with post hoc Tukey or Holm-Šídák test, Mann-Whitney $U$ test, or Kruskal-Wallis test, as specified. Differences between groups were considered significant when $p<0.05$. For all statistical comparisons, ${ }^{*} p<0.05,{ }^{* *} p<0.01{ }^{* * *} p<$ $0.001,{ }^{* * *} p<0.0001$, and NS means not significant. Calculations were performed using GraphPad Prism 7.0 software (GraphPad, La Jolla, CA).

\section{ACKNOWLEDGEMENTS}

We thank N. Power, C. Tu, and M. Noori for their technical assistance. Grant support provided by a Canadian Institute for Health Research Team Grant (THC-13523, D.J.P., K.C.); Ontario Graduate Scholarship in Science and Technology (A.G.); Mount Sinai Hospital Dept. of Medicine Graduate Studentship Award (A.G.) and Postdoctoral Fellowship (W.T., G.Z.); and CIHR Canadian Association of Gastroenterology Fellowship (W.T., G.Z.)

\section{AUTHOR CONTRIBUTIONS}

Conceptualization, A.G. and K.C.; Methodology, A.G., G.Z., D.J.P., and K.C.; Investigation, A.G. and S.R.; Formal analysis, A.G., W.T., and C.J.S.; Writing-original draft, A.G.; Writing-review and editing, G.Z., S.J.R., D.J.P., and K.C.; Funding acquisition, D.J.P. and K.C.; Supervision, D.J.P. and K.C.

\section{ADDITIONAL INFORMATION}

The online version of this article (https://doi.org/10.1038/s41385-018-0128-y) contains supplementary material, which is available to authorized users.

Competing interests: The authors declare no competing interests.

Publisher's note: Springer Nature remains neutral with regard to jurisdictional claims in published maps and institutional affiliations.

\section{REFERENCES}

1. Sender, R., Fuchs, S. \& Milo, R. Revised estimates for the number of human and bacteria cells in the body. PLoS Biol. 14, e1002533 (2016).

2. Lozupone, C. A., Stombaugh, J. I., Gordon, J. I., Jansson, J. K. \& Knight, R. Diversity, stability and resilience of the human gut microbiota. Nature 489, 220-230 (2012).

3. Yatsunenko, T. et al. Human gut microbiome viewed across age and geography. Nature 486, 222-227 (2012)

4. Faith, J. J. et al. The long-term stability of the human gut microbiota. Science $\mathbf{3 4 1}$, 1237439 (2013)

5. Cho, I. \& Blaser, M. J. The human microbiome: at the interface of health and disease. Nat. Rev. Genet. 13, 260-270 (2012).

6. Turpin W., et al. Association of host genome with intestinal microbial composition in a large healthy cohort. Nat. Genet. 48, 1413-1417 (2016).

7. Tamburini, S., Shen, N., Wu, H. C. \& Clemente, J. C. The microbiome in early life: implications for health outcomes. Nat. Med. 22, 713-722 (2016).

8. Marra, F. et al. Antibiotic use in children is associated with increased risk of asthma. Pediatrics 123, 1003-1010 (2009).

9. Scott, F. I. et al. Administration of antibiotics to children before age 2 years increases risk for childhood obesity. Gastroenterology 151, 120-129 (2016).

10. Kronman, M. P., Zaoutis, T. E., Haynes, K., Feng, R. \& Coffin, S. E. Antibiotic exposure and IBD development among children: a population-based cohort study. Pediatrics 130, e794-803 (2012).

11. Shaw, S. Y., Blanchard, J. F. \& Bernstein, C. N. Association between the use of antibiotics in the first year of life and pediatric inflammatory bowel disease. Am. J. Gastroenterol. 105, 2687-2692 (2010).

12. Miyoshi, J. et al. Peripartum antibiotics promote gut dysbiosis, loss of immune tolerance, and inflammatory bowel disease in genetically prone offspring. Cell Rep. 20, 491-504 (2017).
13. Hviid, A., Svanstrom, H. \& Frisch, M. Antibiotic use and inflammatory bowel diseases in childhood. Gut 60, 49-54 (2011).

14. Robertson, S. J. et al. Nod1 and Nod2 signaling does not alter the composition of intestinal bacterial communities at homeostasis. Gut Microbes 4, 222-231 (2013).

15. Segata, N. et al. Metagenomic biomarker discovery and explanation. Genome Biol. 12, R60 (2011).

16. Spor, A., Koren, O. \& Ley, R. Unravelling the effects of the environment and host genotype on the gut microbiome. Nat. Rev. Microbiol. 9, 279-290 (2011).

17. Chassaing, B. et al. Fecal lipocalin 2 , a sensitive and broadly dynamic non-invasive biomarker for intestinal inflammation. PLOS ONE 7, e44328 (2012).

18. Cox, L. M. et al. Altering the intestinal microbiota during a critical developmental window has lasting metabolic consequences. Cell 158, 705-721 (2014).

19. Round, J. L. \& Mazmanian, S. K. Inducible Foxp3+regulatory T-cell development by a commensal bacterium of the intestinal microbiota. Proc. Natl Acad. Sci. USA 107, 12204-12209 (2010).

20. Atarashi, $\mathrm{K}$. et al. $\mathrm{T}_{\text {reg }}$ induction by a rationally selected mixture of Clostridia strains from the human microbiota. Nature 500, 232-236 (2013).

21. Feng, $T$., Elson, C. O. \& Cong, Y. $T_{\text {reg }}$ cell-IgA axis in maintenance of host immune homeostasis with microbiota. Int. Immunopharmacol. 11, 589-592 (2011).

22. Smith, P. M. et al. The microbial metabolites, short-chain fatty acids, regulate colonic $\mathrm{T}_{\text {reg }}$ cell homeostasis. Science 341, 569-573 (2013).

23. Tanoue, T. \& Honda, K. Induction of $\mathrm{T}_{\text {reg }}$ cells in the mouse colonic mucosa: a central mechanism to maintain host-microbiota homeostasis. Semin. Immunol. 24, 50-57 (2012).

24. Round, J. L. \& Mazmanian, S. K. The gut microbiota shapes intestinal immune responses during health and disease. Nat. Rev. Immunol. 9, 313-323 (2009).

25. Dethlefsen, L., Huse, S., Sogin, M. L. \& Relman, D. A. The pervasive effects of an antibiotic on the human gut microbiota, as revealed by deep $16 \mathrm{~S}$ rRNA sequencing. PLoS Biol. 6, e280 (2008).

26. Palleja, A. et al. Recovery of gut microbiota of healthy adults following antibiotic exposure. Nat. Microbiol. 3, 1255-1265 (2018).

27. Dethlefsen, L. \& Relman, D. A. Incomplete recovery and individualized responses of the human distal gut microbiota to repeated antibiotic perturbation. Proc. Nat Acad. Sci. USA 108(Suppl 1), 4554-4561 (2011).

28. Yassour, M. et al. Natural history of the infant gut microbiome and impact of antibiotic treatment on bacterial strain diversity and stability. Sci. Transl. Med. 8 $343 r a 381$ (2016).

29. Chai, G. et al. Trends of outpatient prescription drug utilization in US children, 2002-2010. Pediatrics 130, 23-31 (2012).

30. De Palma, G. et al. Microbiota and host determinants of behavioural phenotype in maternally separated mice. Nat. Commun. 6, 7735 (2015).

31. Moussaoui, N. et al. Chronic early-life stress in rat pups alters basal corticosterone intestinal permeability, and fecal microbiota at weaning: influence of sex. $J$. Neurogastroenterol. Motil. 23, 135-143 (2017).

32. Bokulich, N. A. et al. Antibiotics, birth mode, and diet shape microbiome maturation during early life. Sci. Transl. Med. 8, 343ra382 (2016).

33. Russell, S. L. et al. Early life antibiotic-driven changes in microbiota enhance susceptibility to allergic asthma. EMBO Rep. 13, 440-447 (2012).

34. Couturier-Maillard, A. et al. NOD2-mediated dysbiosis predisposes mice to transmissible colitis and colorectal cancer. J. Clin. Invest. 123, 700-711 (2013).

35. Petnicki-Ocwieja, T. et al. Nod2 is required for the regulation of commensal microbiota in the intestine. Proc. Natl Acad. Sci. USA 106, 15813-15818 (2009).

36. Ramanan, D., Tang, M. S., Bowcutt, R., Loke, P. \& Cadwell, K. Bacterial sensor Nod2 prevents inflammation of the small intestine by restricting the expansion of the commensal Bacteroides vulgatus. Immunity 41, 311-324 (2014).

37. Stappenbeck, T. S. \& Virgin, H. W. Accounting for reciprocal host-microbiome interactions in experimental science. Nature 534, 191-199 (2016).

38. Kobayashi, K. S. et al. Nod2-dependent regulation of innate and adaptive immunity in the intestinal tract. Science 307, 731-734 (2005).

39. Jing, X. et al. Peptidoglycan recognition protein 3 and Nod2 synergistically protect mice from dextran sodium sulfate-induced colitis. J. Immunol. 193, 3055-3069 (2014).

40. Korpela, K. et al. Intestinal microbiome is related to lifetime antibiotic use in Finnish pre-school children. Nat. Commun. 7, 10410 (2016).

41. Azad, M. B., Bridgman, S. L., Becker, A. B. \& Kozyrskyj, A. L. Infant antibiotic exposure and the development of childhood overweight and central adiposity. Int. J. Obes. 38, 1290-1298 (2014).

42. Schulfer, A. F. et al. Intergenerational transfer of antibiotic-perturbed microbiota enhances colitis in susceptible mice. Nat. Microbiol. 3, 234-242 (2018).

43. Sellon, R. K. et al. Resident enteric bacteria are necessary for development of spontaneous colitis and immune system activation in interleukin-10-deficient mice. Infect. Immun. 66, 5224-5231 (1998). 
44. Moran, C. J. et al. IL-10R polymorphisms are associated with very-early-onset ulcerative colitis. Inflamm. Bowel Dis. 19, 115-123 (2013).

45. Russell, S. L. et al. Perinatal antibiotic treatment affects murine microbiota, immune responses and allergic asthma. Gut Microbes 4, 158-164 (2013).

46. Lochner, M. et al. In vivo equilibrium of proinflammatory IL-17+and regulatory IL10+Foxp3+RORgamma t+T cells. J. Exp. Med. 205, 1381-1393 (2008).

47. Lochner, M. et al. Restricted microbiota and absence of cognate TCR antigen leads to an unbalanced generation of Th17 cells. J. Immunol. 186, 1531-1537 (2011).

48. Solomon, B. D. \& Hsieh, C. S. Antigen-specific development of mucosal Foxp3 + RORgammat $+\mathrm{T}$ cells from regulatory $\mathrm{T}$ cell precursors. J. Immunol. 197, 3512-3519 (2016).

49. Yang, B. H. et al. Foxp3(+) T cells expressing RORgammat represent a stable regulatory T-cell effector lineage with enhanced suppressive capacity during intestinal inflammation. Mucosal Immunol. 9, 444-457 (2016).

50. Schiering, $C$. et al. The alarmin IL-33 promotes regulatory T-cell function in the intestine. Nature 513, 564-568 (2014).

51. Wang, Y., Su, M. A. \& Wan, Y. Y. An essential role of the transcription factor GATA3 for the function of regulatory T cells. Immunity 35, 337-348 (2011).
52. Wohlfert, E. A. et al. GATA3 controls Foxp3(+) regulatory T cell fate during inflammation in mice. J. Clin. Invest. 121, 4503-4515 (2011).

53. Ohnmacht, C. et al. MUCOSAL IMMUNOLOGY. The microbiota regulates type 2 immunity through RORgammat(+) T cells. Science 349, 989-993 (2015).

54. Caporaso, J. G. et al. Ultra-high-throughput microbial community analysis on the Illumina HiSeq and MiSeq platforms. ISME J. 6, 1621-1624 (2012).

55. Masella, A. P., Bartram, A. K., Truszkowski, J. M., Brown, D. G. \& Neufeld, J. D. PANDAseq: paired-end assembler for illumina sequences. BMC Bioinformatics 13, 31 (2012).

56. Caporaso, J. G. et al. QIIME allows analysis of high-throughput community sequencing data. Nat. Methods 7, 335-336 (2010).

57. Edgar, R. C. Search and clustering orders of magnitude faster than BLAST. Bioinformatics 26, 2460-2461 (2010).

58. DeSantis, T. Z. et al. Greengenes, a chimera-checked $16 \mathrm{~S}$ rRNA gene database and workbench compatible with ARB. Appl. Environ. Microbiol. 72, 5069-5072 (2006). 